\title{
Characteristics of a bridge condition assessment method based on state representation methodology (SRM) and damage detection sensitivity
}

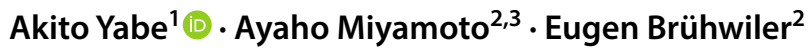

Received: 25 November 2018 / Accepted: 27 February 2019 / Published online: 12 March 2019

(c) The Author(s) 2019

\begin{abstract}
The State Representation Methodology (SRM) combined with the Frequency Slice Wavelet Transform (FSWT), which is a new time-frequency analysis tool, is proposed for assessing the condition of bridges on the basis of bridge monitoring data. First, this paper presents an overall description of the SRM method via FSWT analysis. It then shows, through numerical simulations, some novel characteristics and advantages of FSWT analysis in contrast to the conventional wavelet approach and the feature extraction accuracy of SRM analysis for the detection of bridge damage on the basis of monitoring data. The principal results obtained through this study can be summarized as follows: (1) details of a newly proposed SRM and its application to bridge condition assessment based on bridge monitoring data are introduced. The proposed SRM combined with the FSWT is validated as a novel time-frequency analysis tool for assessing bridge condition on the basis of bridge monitoring data. (2) New properties of FSWT analysis are demonstrated, and advantages in contrast to the traditional wavelet method are highlighted. Feature extraction in SRM analysis is precise for damage detection in a bridge system on the basis of monitoring data and using numerical simulations.
\end{abstract}

Keywords Bridge health monitoring · Bridge condition assessment - Damage detection - State representation methodology $(\mathrm{SRM}) \cdot$ Extracting accuracy

\section{Introduction}

Various structural health monitoring (SHM) approaches have been proposed with the aim of facilitating the maintenance of existing bridges, and many examples of such systems have been reported [1]. The implementation of a practical SHM system requires the integration of a number of technologies related to sensing, instrumentation and telecommunications.

Akito Yabe

akito-yabe@kke.co.jp

Ayaho Miyamoto

miya818@yamaguchi-u.ac.jp

Eugen Brühwiler

eugen.bruehwiler@epfl.ch

1 Sustainable Solutions Department, Kozo Keikaku Engineering Inc, Tokyo, Japan

2 Swiss Federal Institute of Technology (EPFL), Lausanne, Switzerland

3 Emeritus of Yamaguchi University, Yamaguchi, Japan
SHM features extraction (damage detection) techniques and performance evaluation and diagnosis methods. The accumulation, over a long period, of measurement data related to the bridge of interest is of particular importance. Such data include environmental conditions, such as temperature and humidity, as well as physical quantities directly related to structural characteristics, such as vibration, strain and displacement characteristics. These data are then used for estimating the present health status and future deterioration (damage) of the bridge [2-8]. Lifetime engineering based on monitoring data obtained from bridge structures is becoming one of the most important issues worldwide [2,3]. Also, it is important to detect damage from a large amount of monitoring data. Therefore, discovering damage information of a target bridge is a big challenge [4-8].

Figure 1 shows the importance of condition assessment in SHM research. This figure reveals that practical condition assessment is still a basic problem in SHM research, although there is rich literature related to the topic of SHM. Focusing on damage and the vibration characteristics of bridge members, the authors have already proposed 


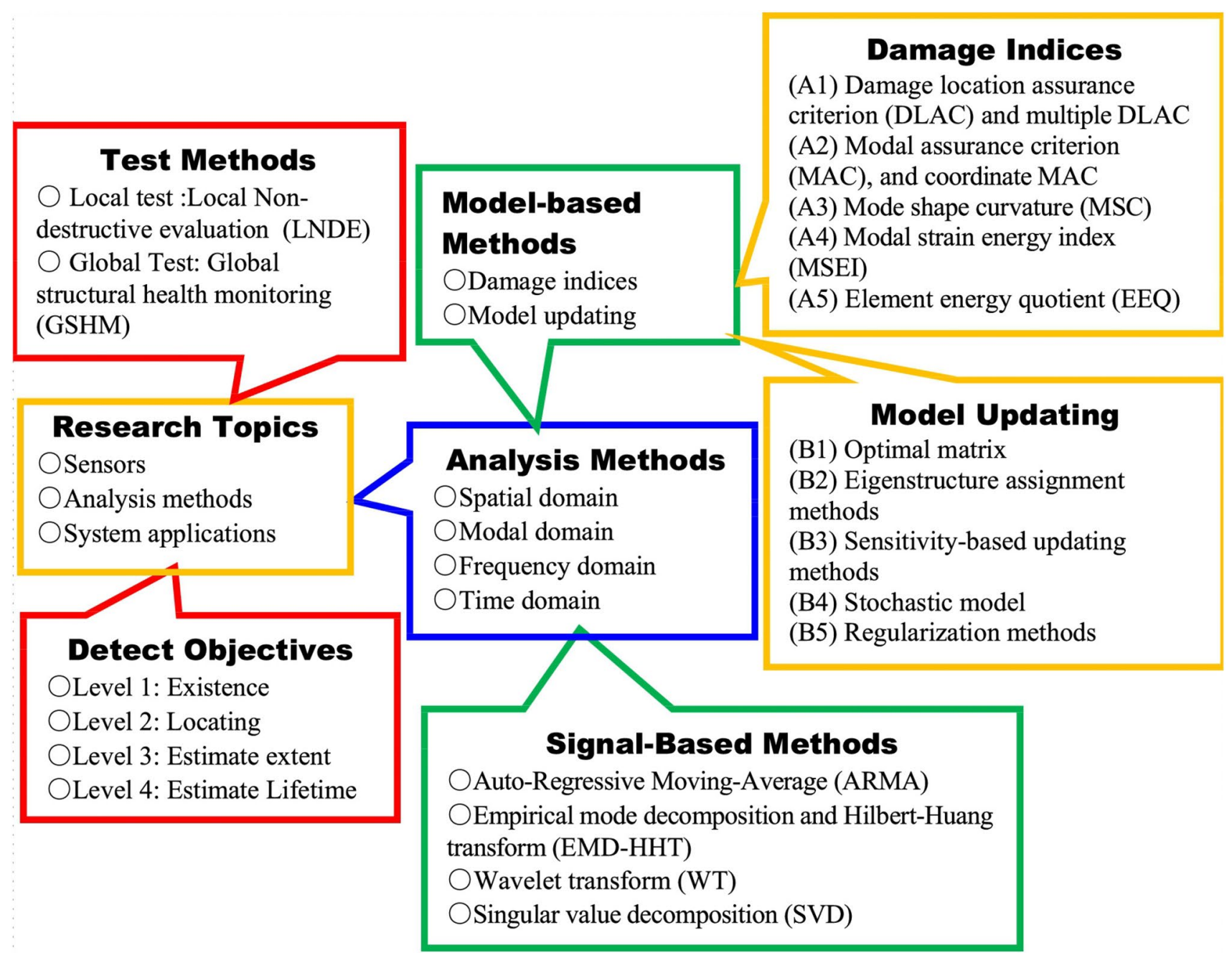

Fig. 1 Importance of condition assessment in SHM

an analysis technique called the Frequency Slice Wavelet Transform (FSWT) as a time-frequency analysis technique suitable for the preprocessing of large amounts of monitoring data $[9,10]$. The authors have also developed the State Representation Methodology (SRM), a new technique for detecting damage by constructing feature vectors based on analytical results obtained from FSWT analysis [11, 12]. Figure 2 shows the procedure for detecting damage by expressing a damage-induced change with an SRM-based probabilistic model. Since there are many methods that can realize a monitoring data collection system, the first step of damage detection using SRM is related to the choice of sensor types, optimum sensor locations for damage detection, the big data collection system, etc. for the target structure to be monitored.

This paper discusses the characteristics and validity of the FSWT, which is a tool used for SRM-based probabilistic modeling, by comparing it with conventional methods. For example, the traditional methods based on frequency domain decomposition (FDD) are widely recognized as being simple; however, they tend to often lead to lower sensitivity and accuracy of damage detection, because the power spectrum of the measured responses cannot be accurately estimated, particularly for highly damped systems and systems with severe modal interference and high noise. Meanwhile, there are some important questions with these approaches: The natural frequency, corresponding mode shape and damping coefficient usually change very slowly when a bridge system is deteriorating in an early stage; in other words, the bridge health condition is not always so sensitive with those parameters. At the same time, since a complex system includes many structural parts, it has many frequency components, and they interfere with each other. It is thus difficult to use the FDD method to analyze a system in damage detection because it is impossible to get modal parameters exactly. It is necessary to use an overall view to recognize a system. One can assume that the system state is described by a certain (non-parametric) dynamic variable that could be described 


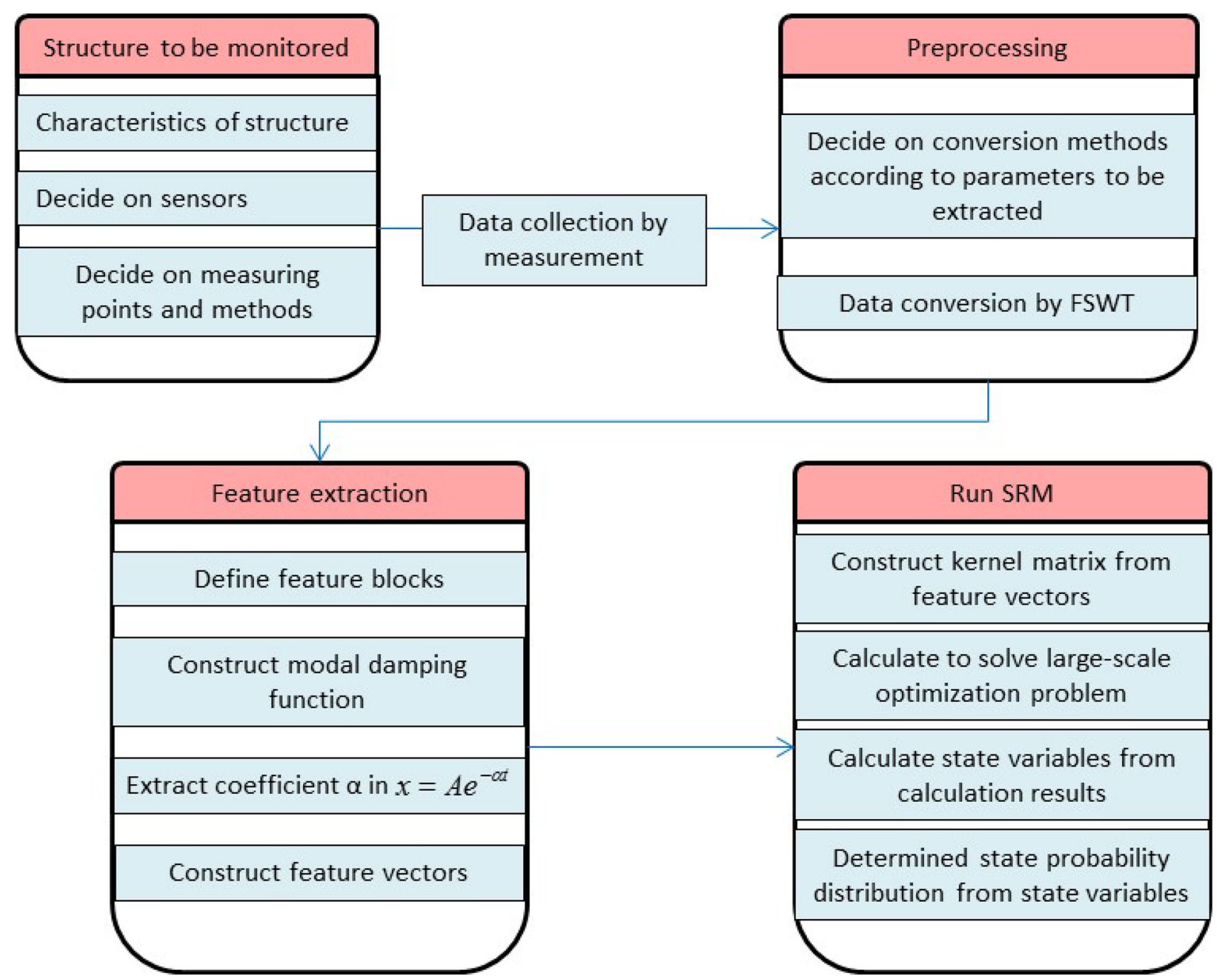

Fig. 2 Procedure for damage detection by use of SRM

by a certain mathematical model, and a probability-based method would be better for describing its state because it contains many random factors.

In this study, we used the test results obtained from two types of experiments, namely, impact hammer tests and moving load experiments on simulated bridge girders with artificially introduced damage. Also, we discuss the characteristics of the SRM and FSWT as performed by following the procedures shown in Fig. 2 and considering operational conditions associated with scale parameters and other factors affecting damage detection accuracy.

\section{Characteristics of the SRM-FSWT method}

This section gives an outline of the basic concept of the nonparametric description of a structural system's state, called the State Representation Methodology (SRM). It focuses on the SRM idea and its application to bridge condition assessment based on bridge health monitoring data.

The maintenance of existing bridges on the basis of life cycle cost (LCC) has become an important research area in many countries worldwide. Therefore, efforts are underway in many (mostly industrialized) countries to develop and put into practical use various bridge management systems (BMSs) and structural health monitoring (SHM) systems. BMS-assisted structural health evaluation and remaining life estimation of bridges rely mainly on engineering judgment based on data obtained from periodic visual inspections of the bridges. In such evaluation or estimation, the degree of deterioration is estimated from deterioration curves specified for different deterioration mechanisms on the basis of accumulated inspection data. If future deterioration is to be predicted, however, two conditions need to be met. One is that the deterioration mechanism must be known, and the other is that the progress of deterioration must be predictable. It 
cannot be denied, therefore, that the proposed method may give erroneous predictions under certain circumstances, such as when a number of factors interact with one another in a complex manner, or when damage has been caused by invisible factors (see, for example, Fig. 3).

Depending on the monitoring items of the bridge of interest, such as static deflection and dynamic displacement and acceleration, the SHM-assisted management approach also poses a number of problems in distinguishing between changes that directly affect the degree of progress

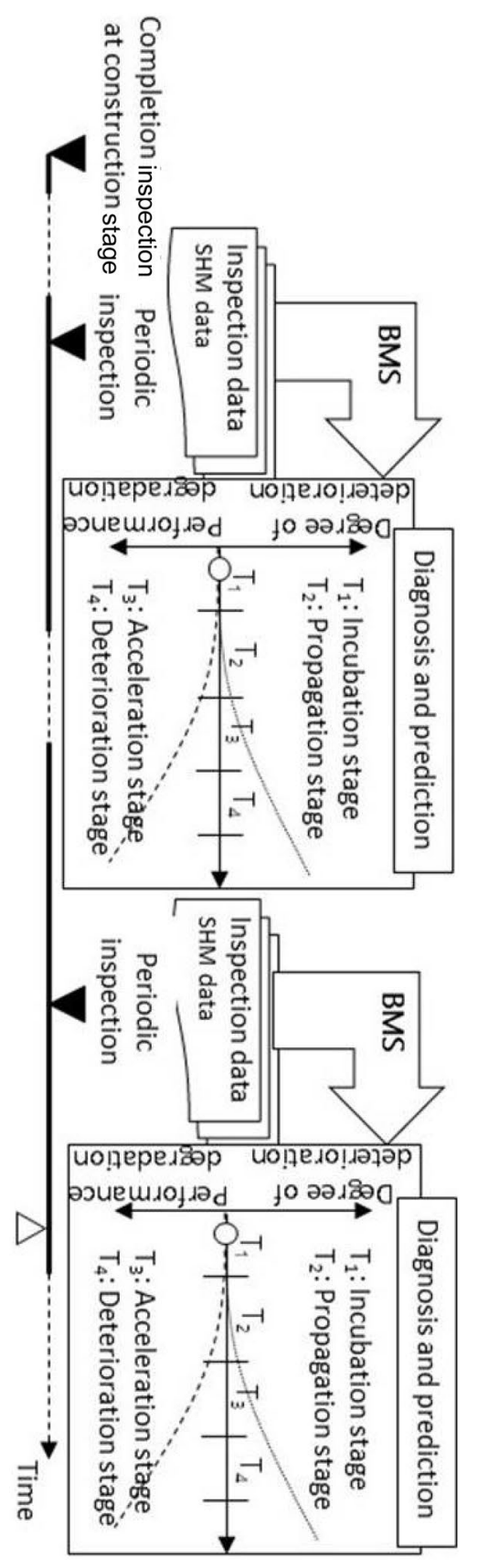

Fig. 3 Example of structural health diagnosis by conventional method of deterioration and changes that do not, from among a large amount of collected data. There is as yet no established method, for evaluating the structural soundness of bridges with sufficient accuracy with the assistance of structural health monitoring alone. As a practical approach, it is necessary to make effective use of the conventional BMS approach in conjunction with SHM. If SHM is used as an aid, the amount of information that needs to be handled is far greater than in cases where a conventional approach, such as visual inspection, is used. In such cases, it is of critical importance, although technologically challenging, to identify useful data among the accumulated monitoring data and extract relevant information efficiently [11].

Focusing on SRM, which is a newly developed method for rationally evaluating the present state (performance) of a structural system by analyzing a large amount of measurement data obtained continuously from a network of SHM system sensors and extracting useful information, this study evaluates the characteristics of SRM using bridge model girders. The SRM in this study uses the Frequency Slice Wavelet Transform (FSWT), which is a time-frequency analysis technique suitable for the preprocessing of a large amount of monitoring data, as a foundation on which to establish a new damage detection method that pays attention to different types of damage to bridge members and their vibration characteristics. By applying a modal damping function (MDF) to the analytical results thus obtained, amplitude envelope coefficients are extracted, and feature vectors are constructed. The proposed method is a new damage detection technique developed using the feature vectors constructed through the extraction process mentioned above. The proposed method makes it possible to distinguish between changes in data caused directly by deterioration and other changes by checking on the constantly changing likelihood of feature quantities determined by SRM relative to time series monitoring data obtained by SHM, as well as structural health evaluation data obtained from the BMS. Figure 4 illustrates the process of structural health diagnosis by the proposed method.

\subsection{Concept of state representation methodology (SRM)}

The state of a system is interpreted as the overall response to its internal and external factors, which essentially depends on the response of its structure or structural properties and natural environment. The quantitative expression of the system state is the description of the system responding to incentive factors. If the response satisfies our expectation, the system state is considered as a normal state, and otherwise as an abnormal state. In usual circumstances or under normal use conditions, the system is in a stable state, which means that its state is constant, or generally fluctuates in the vicinity of a steady state. 


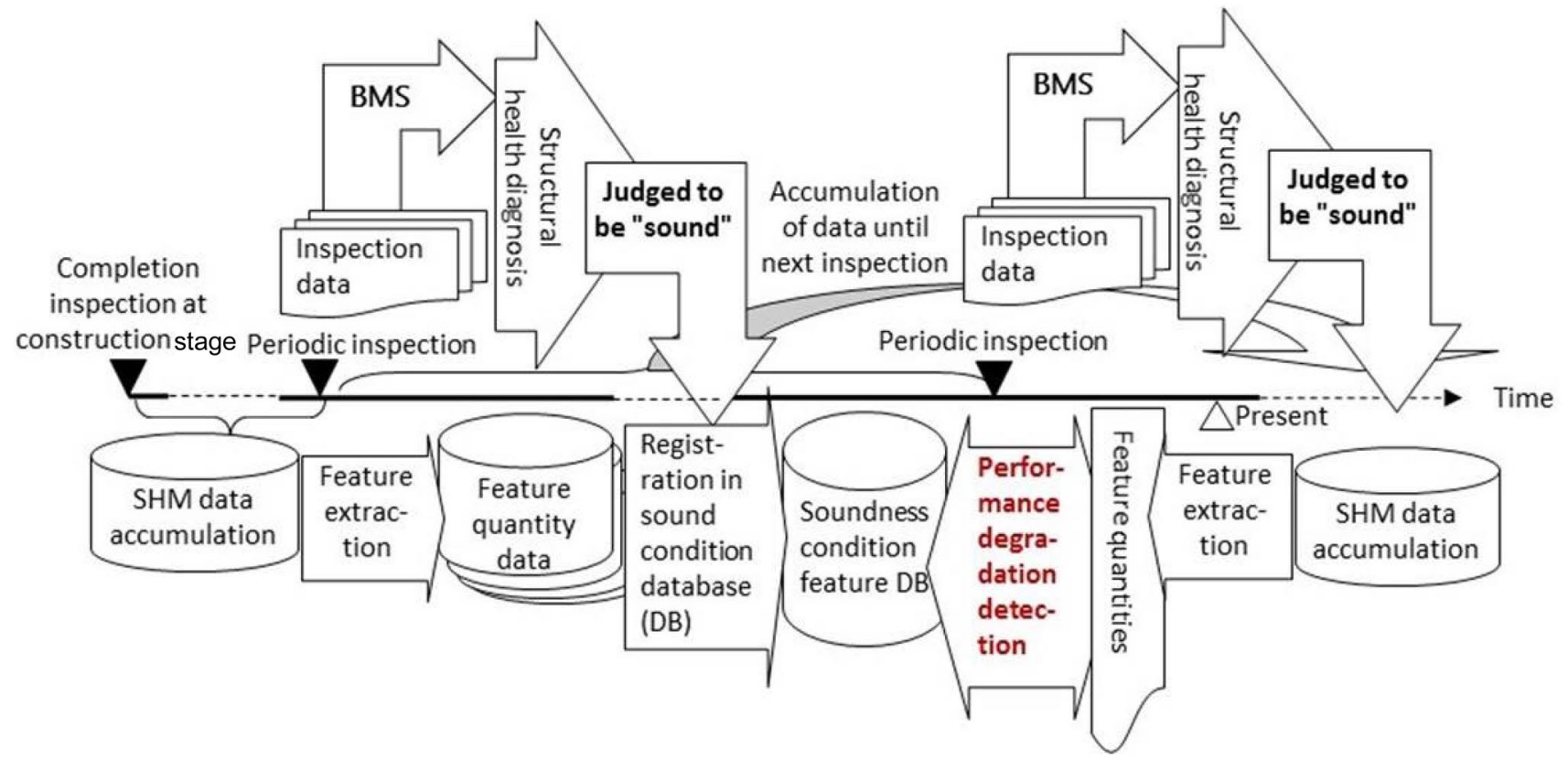

Fig. 4 Flow of structural health diagnosis by the proposed method and what it is supposed to do

Therefore, usually it is assumed that the state it is a steady random variable that often follows the normal distribution.

\subsubsection{System state representation}

A basic vibration equation as a parameterized example is:

$M \delta^{\prime \prime}+C \delta^{\prime}+K \delta=0$

Although it is easy to determine the parameters $M, C$, and $K$ for a single degree of freedom ( $\mathrm{S}-\mathrm{DOF}$ ) system, in general, it is not easy to determine the parameters for a multi-DOF system, because $M, C$, and $K$ are correlated within the system characteristics. From the vibration equation for an S-DOF system, the vibration frequency $\omega$ can be written as:

$\omega^{2}=\frac{K}{M}-\frac{C^{2}}{4 M^{2}}$

This can also be written as:

$M=\frac{K \pm \sqrt{K^{2}-(\omega C)^{2}}}{2 \omega^{2}}$ or $M=f(K, \omega, C)$

On the other hand, for a multi-DOF system, it can be written as:

$\sum_{i} f\left(K_{i}, \omega_{i}, C_{i}\right)=M_{\mathrm{total}}=$ CONSTANT

or

$\sum_{i} \frac{1}{M_{\text {total }}} f\left(K_{i}, \omega_{i}, C_{i}\right)=1 ;$ CONSTANT
In this state, let us apply the Taylor formula to expand the function $f\left(K_{i}, \omega_{i}, C_{i}\right)$ to obtain:

$\sum_{i} A_{i}\left(K_{i}, C_{i}\right) \omega_{i}+o\left(\omega_{1}, \omega_{2}, \ldots \omega_{m}\right)=1$

This equation can be written more generally as:

$\sum_{i} \alpha_{i} \omega_{i}+o\left(\omega_{1}, \omega_{2}, \ldots \omega_{m}\right)=1$

If the state of the vibration system is denoted by the variable $\zeta$ then:

$\zeta=\sum_{i} \alpha_{i} \omega_{i}+o\left(\omega_{1}, \omega_{2}, \ldots \omega_{m}\right)=1$

If the system is not changed, $\zeta$ is a constant value, and it is expressed as an implicit function of the system feature parameters. In an actual existing bridge system, the parameters $M, K$, and $C$ are not accurately known; however, it is relatively easy to know the response parameters $\left(\omega_{1}, \omega_{2}, \ldots \omega_{n}\right)$ from sensors. Then, the question is how to establish a function including the state variable $\zeta$ with $\left(\omega_{1}, \omega_{2}, \ldots \omega_{n}\right)$ ? These functions are called the State Representation Equation (SRE) or State Representation Function (SRF) of the system.

The following section will give more details of a SRE that can be approximated by a linear or nonlinear combination of system features [12]. 


\subsubsection{State representation equation (SRE)}

There are a lot of responses in a complex structural system such as a bridge, that are involved in test data or experimental data. It is impossible to obtain all of the system features from data as obtained by a finite number of sensors. Therefore, it is assumed that the system state of a complex system is a function of the system state space. A projector or a part of the system state can be revealed by means of limited observation, which will be possible to realize in an actual application. Naturally, at the current time, all of the system responses are considered as the system state space or feature space, denoted as $H^{\infty}$, i.e.,

$\left(h_{1}(t), h_{2}(t), h_{3}(t), \ldots h_{n}(t) \ldots\right) \in H^{\infty}$

where each $h_{i}(t)$ is a projector of a system feature, called a system sub-response function. For example, in an experiment, if the input to a system having many responses is an excitation signal $r_{i}(t)$, the output signal, $s_{i}(t)$ as the test result, can be obtained by the sub-response function $h_{i}(t)$ using the following methods in the time domain:

$s_{i}(t)=\int_{-\infty}^{\infty} r_{i}(\tau) h_{i}(t-\tau) \mathrm{d} \tau, \quad i=1,2,3 \ldots \infty$

In the frequency domain, it becomes:

$S_{i}(\omega)=R_{i}(\omega) H_{i}(\omega), i=1,2,3 \ldots \infty$

Then, the sub-responses are:

$H_{i}(\omega)=\frac{S_{i}(\omega)}{R_{i}(\omega)}, i=1,2,3 \ldots \infty$

Usually, $h_{i}(t)$ is independent of $r_{i}(t), i=1,2,3, \ldots \infty$.

Therefore, in SRM, for an objective system $S$, a nonobjective condition state variable, $\zeta$, is defined as $\zeta \in[0,1]$ or $\zeta \in(0,1)$, which is a function of its state space:

$\zeta=f\left(h_{1}(t), h_{2}(t), h_{3}(t), \ldots h_{n}(t) \ldots\right)$

This equation can be changed into the frequency domain:

$\zeta=f\left(H_{1}(\omega), H_{2}(\omega), \ldots H_{n}(\omega) \ldots\right)$

Here, the main object in SRM is focused on the relationship between the system responses and its system state (feature), $\zeta$, in the system feature space $H^{\infty}$. The SRM describes the system state and some system assessment methods when the system conditions have been changed.

In the SRM, if one regards the current system state as the normal state, this means that the state is subjected to expectation, as with safety, reliability etc., which can be viewed as state (feature) $\zeta=1$, i.e.:

$\zeta=f(\cdot) \equiv 1$, if the system is always in the normal state

With the passage of time, the system exhibits some gradual deterioration in the structures, such as bridge, and it will depart from the normal state; in a general sense, this is called the deterioration state and it can be assumed to be as follows:

$\zeta=f(\cdot) \leq 1$, always

In a complex system, it is very difficult to test the exact condition state with sensors. Therefore, the lowest level of state, herein noted $c_{0}$, was taken into consideration. At the same time, by means of a test, part of its responses to the environment, $c_{0}$, can then be estimated and represented using the following inequality:

$\zeta=f(\cdot) \geq c_{0}$

Figures 2 and 8 show a conceptual procedure of the feature extraction by the above-mentioned SRM, where $x$ is the system feature vector, $\zeta$ is the state variable, and $\lambda$ is the system structure alias parameter.

\subsection{Damage detection method based on SRM}

\subsubsection{Basic idea of state representation}

1. Extraction of a finite-dimension feature subspace to approximate the state function, $f$

Because the real representation function, $f$, is unknown, some properties related to the relationships between their features and the condition state may be correlated to be approximated by a method (what kinds of features). In fact, $f$ can be approximated by some mathematical expressions. The successful use of SRM is dependent upon the experimenter's ability to develop a suitable approximation for $f(\cdot)$.

Note: $H^{n}$ is a finite-dimension subspace such that $H^{n} \subset H^{\infty}$ and state variable, $\zeta$, can be limited on $H^{n} \subset H^{\infty}$, i.e., $\zeta=f(h), h \in H^{n} \subset H^{\infty}$.

\section{Standardization of feature vector}

Let $\quad h \in H^{n} \subset H^{\infty}, \quad h:=\frac{h}{\|h\|}$

It is necessary to standardize the unit representation of feature vector, $h$, because of the non-parametric description.

3. Find system state support vector

Let us assume system feature vector, $x:=(x(1), x(2), \ldots x(n)) \in H^{n}$. If a constant vector $w \in R^{n}$ called the system state support vector or feature director exists, then it needs to satisfy the following relation (equation):

$$
1=\langle w, x\rangle=\sum_{k=1}^{n} w(k) x(k)
$$


This is the first-order model of state $\zeta$ and is given by:

$\zeta=f(w, x)=\sum_{k=1}^{n} w(k) x(k)$

If the system has only one response vector signal $h \in H^{n}$, the solution using the Least Squares Estimators method should be: $w=h$. However, if it is a multiple response vector, then let

$w_{i}=h_{i}, i=1,2 \ldots m$

Now let every response vector $h \in H^{n}$ be a projector of the current system state along with its feature directors $w_{i}, i=1,2 \ldots m$ [15], and it has a weight: $\lambda_{i} \geq 0$ and

$\lambda_{i} \in(0,1), \sum_{i=1}^{m} \lambda_{i}=1, \quad w=\sum_{i=1}^{m} \lambda_{i} w_{i}$

Then,

$1=\langle w, x\rangle=\left\langle\sum_{i=1}^{m} \lambda_{i} h_{i}, x\right\rangle=\sum_{i=1}^{m} \lambda_{i}\left\langle h_{i}, x\right\rangle$

In general, the state function includes parameter vector, $\alpha$, which is written as:

$f(\alpha, x)=\sum_{i=1}^{m} \alpha_{i}\left\langle h_{i}, x\right\rangle$

The function $f$ is a linear operator, and the vector $\alpha$ is herein called a system state parameter.

4. Modify the product relation for feature vectors by means of Kernel Function Method

Since the representation of a system state with a first-order linear model is limited, it is a very useful idea to use a kernel function method to solve a high-order nonlinear representation of the system state.

A Kernel Function Map, $x \rightarrow \varphi(x)$, is defined herein as a nonlinear function that changes from the current variable space into another parameter space, which can practically be understood as a transformation for the sensor's function. Then, we redefine $\langle s, x\rangle, s \in H^{n}, x \in H^{n}$ as,

$\langle s, x\rangle=\langle\varphi(s), \varphi(x)\rangle=k(s, x), s \in H^{n}, x \in H^{n}$

Here, $k(s, x)=\langle\varphi(s), \varphi(x)\rangle$ is a kernel function. There are many choices for the kernel function, $k(\cdot, \cdot)$.

In the present paper, the kernel functions recommended in Ref. [15] are used:

$k(s, x)=\exp \left(-\frac{d(s, x)}{\sigma^{2}}\right)$, where, $\sigma$ is the SRM scale.
Here, $d(s, x)=\sum_{i=1}^{n} \frac{\left(s_{i}-x_{i}\right)^{2}}{s_{i}+x_{i}}$ or $d(s, x)=\left(\sum_{i=1}^{n}\left|s_{i}-x_{i}\right|^{p}\right)^{1 / p}$

$k(s, x)=(s \cdot x)^{d}, \quad(\|s\|=1,\|x\|=1)$

Let us define $\Omega=\sum_{k=1}^{m} \lambda_{k} k\left(h_{k},.\right)$ as an operator, called the system state support operator or the system state representation operator, which is a highly nonlinear operator. It is important to find a support operator to represent and assess the system state.

\section{Computing support vector}

Take a train set of system feature vectors represented by $T S$.

Define the Gram matrix : $G=\left(\begin{array}{c}k\left(x_{1}, x_{1}\right), k\left(x_{1}, x_{2}\right), \ldots k\left(x_{1}, x_{m}\right) \\ k\left(x_{2}, x_{1}\right), k\left(x_{2}, x_{2}\right), \ldots k\left(x_{2}, x_{m}\right) \\ \ldots \\ k\left(x_{m}, x_{1}\right), k\left(x_{m}, x_{2}\right), \ldots k\left(x_{m}, x_{m}\right)\end{array}\right)$

$$
e=(1,1, \ldots 1)^{\mathrm{T}} \text {. }
$$

$\min \left\|\left(I-\frac{1}{n} e e^{T}\right) G \lambda\right\|^{2}$

Subject to $\sum_{i} \lambda_{i}=1,0 \leq \lambda_{i} \leq 1, i=1,2,3, \ldots m$

It follows that:

$\zeta=f(\lambda, x)=\sum_{i}^{m} \lambda_{i} k\left(x_{i}, x\right)$

\subsubsection{SRM algorithm}

The SRM algorithm for computing the state variable, $\zeta=f(\lambda, x)$ or $\zeta=f(\alpha, x)$ using Eq. (28) with a kernel function such as Eqs. (24), (26), etc. is shown here for linear or quadratic convex programming, which are similar to the familiar Support Vector Machine (SVM) methods. An example is shown below in the form of steps to make it more clear and easy to understand:

STEP 1: Input: $\left(x_{1}, x_{2}, \ldots x_{m}\right), x_{i} \in H^{n}$, matrix $A$ from Eq. (28), iteration number $N$, and error $\varepsilon$.

STEP 2: Set initial point, $x=e / n, D_{0}=\operatorname{diag}\left(\lambda_{0 i}\right)$.

STEP 3: $k=0$.

STEP 4: while $k<N$, do:

STEP 5: Set $D_{k}=\operatorname{diag}\left(\lambda_{k i}\right)$.

STEP 6: Compute search direction, $p_{k}$, such as, set $p_{k}=D_{k} A^{T} A D_{k} e$. 
STEP 7: Compute optimal search step $t$ to satisfy $\min _{t p<e}\left\|A D_{k}\left(e-t p_{k}\right)\right\|^{2}$, and set $\lambda_{k+1}=\lambda_{k}-t D_{k} p_{k}$.

STEP 8: if $\left|\lambda_{k+1}-\lambda_{k}\right| \leq \varepsilon$, break and go to STEP 11 .

STEP 9: set $k=k+1$; go to STEP 4 .

STEP 10: end while.

STEP 11: stop.

\subsubsection{Damping feature extracting algorithm}

The features of a damping vibration signal include its frequency and damping coefficient. It is usually not easy to get the damping coefficient exactly because, in general a damping vibration signal always includes many interference frequencies or noise. In this paper, it is assumed that the envelope curve of a damping vibration signal is: $x=A e^{-\alpha t}$ as obtained by FSWT time-frequency transformation. Therefore, we can get the transient damping coefficient at any frequency and at any time based on FSWT [12].

\section{Time-frequency space analysis method in SRM}

In this study, with the aim of extracting damping-related information multi-dimensionally, damping parameters are extracted from the time-frequency space, and the amount of change in each damping parameter under the influence of changing vibrations is taken into consideration. Beside the FSWT [Eq. (30) shown below] mentioned earlier, methods for performing time-frequency space analysis from vibration data as shown in Fig. 5 include the short-time Fourier transform (STFT) using Eq. (31), the continuous wavelet transform (CWT) using Eq. (32) and the Wigner-Ville distribution (WVD) using Eq. (33) [13, 14].

However, no definite study results have been provided that show which of the above-mentioned methods is more resistant to noise in the vibration data to be analyzed compared with other methods and which method is more suitable for use in structural health diagnosis.

$W_{f}(t, \omega, \kappa)=\frac{1}{2 \pi} \int_{-\infty}^{+\infty} \hat{f}(u) \hat{p}^{*}\left(\kappa \frac{u-\omega}{\omega}\right) e^{i u t} \mathrm{~d} u$

where $\hat{f}(u)$ is Fourier transport, and $\hat{p}(\omega)$ is the Frequency slice function (FSF).

$F(\omega, b)=\int_{-\infty}^{\infty} f(t) w(t-b) e^{-j \omega t} \mathrm{~d} t$

where $w$ is a window function.

$T(a, b)=\int_{-\infty}^{+\infty} x(t) \psi_{a, b}^{*}(t) \mathrm{d} t$ where $\psi(t)$ is a mother wavelet.

$P(t, w)=2 \int_{-\infty}^{\infty} s(t+\tau) s^{*}(t-\tau) e^{-j 2 \omega t} \mathrm{~d} \tau$

where the asterisk means the complex conjugate.

This study, therefore, shows that the FSWT is more suitable for SRM than the other time-frequency space analysis methods mentioned above from the viewpoint of resistance to noise. Meanwhile, the effect of the characteristics of variance $\sigma$ (SRM scale parameter) on detection accuracy in cases where a Gaussian kernel is used in SRM-based probabilistic modeling is discussed.

\section{Noise-related characteristics of different time-frequency space analysis methods}

Figure 6 shows the differences in time-frequency space analysis results obtained using two types of sample vibration data. The basic sample vibration was defined using the wave function expressed by Eq. (34) (see Fig. 6a):

$s\left(t, A, f, \varsigma, \theta, t_{0}\right)=\left\{\begin{array}{cc}A e^{-2 \pi f \varsigma t} \cos \left(2 \pi f_{d} t+\theta\right) & t \geq t_{0} \\ 0 & t<t_{0}\end{array}\right.$

where $s=s_{1}+s_{2}+s_{3}+s_{4}$. This wave is a simulated 10-second wave derived using the settings shown in Table 1.

Figure 6f shows a simulated wave obtained by adding $25 \%$ white noise to Fig. 6a. Figure 6b, g shows scalogram calculation results for Fig. $6 \mathrm{a}$, f, respectively, obtained using the FSWT. Similarly, Fig. 6c, h shows the results obtained using the STFT; Fig. 6d, i, the results obtained using the CWT (Morlet); and Fig. 6e, j, the results obtained using the WVD. Each figure also shows the parameters (e.g., scale) used in the analysis.

First, the analytical results in Fig. 6b, c show that for the frequencies (signals) of $15 \mathrm{~Hz}, 35 \mathrm{~Hz}$, and $50 \mathrm{~Hz}$ (twice) produced from the simulated wave, the CWT (Morlet)-based scalogram clearly shows their peaks in time-frequency space but is slightly inferior in peak clarity to the FSWT-based scalogram. Comparison of Fig. $6 \mathrm{~g}$ and h reveals that the CWT (Morlet)-based scalogram has lost clarity because of the noise, indicating that obtaining clear peaks is difficult.

Next, comparison of Fig. $6 \mathrm{~b}$ and d reveals that the analytical results obtained using the STFT are better in clarity than the results obtained using the CWT (Morlet), and are almost as good as the results obtained using the FSWT. Comparison of Fig. $6 \mathrm{~g}$ and i reveals that, like the analytical results obtained using the CWT (Morlet), the STFT-based scalogram loses clarity because of noise, indicating that extracting clear peaks is difficult. 
Fig. 5 Examples of results of time-frequency space analysis of vibration data and their comparison $[9,10]$
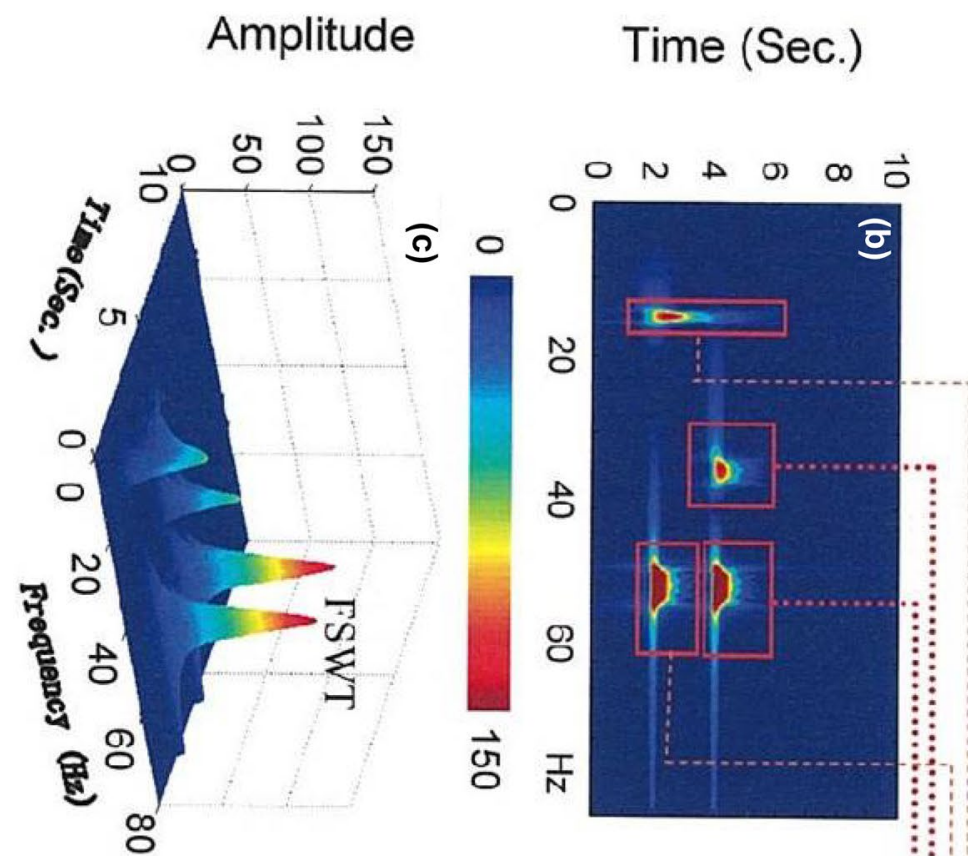

\section{Simulated Signal}

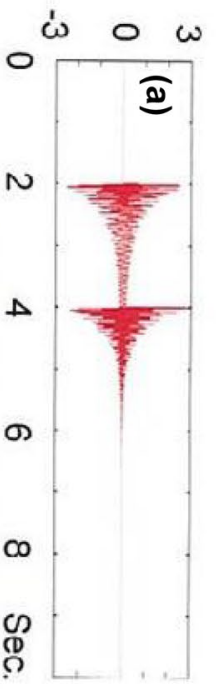

FT
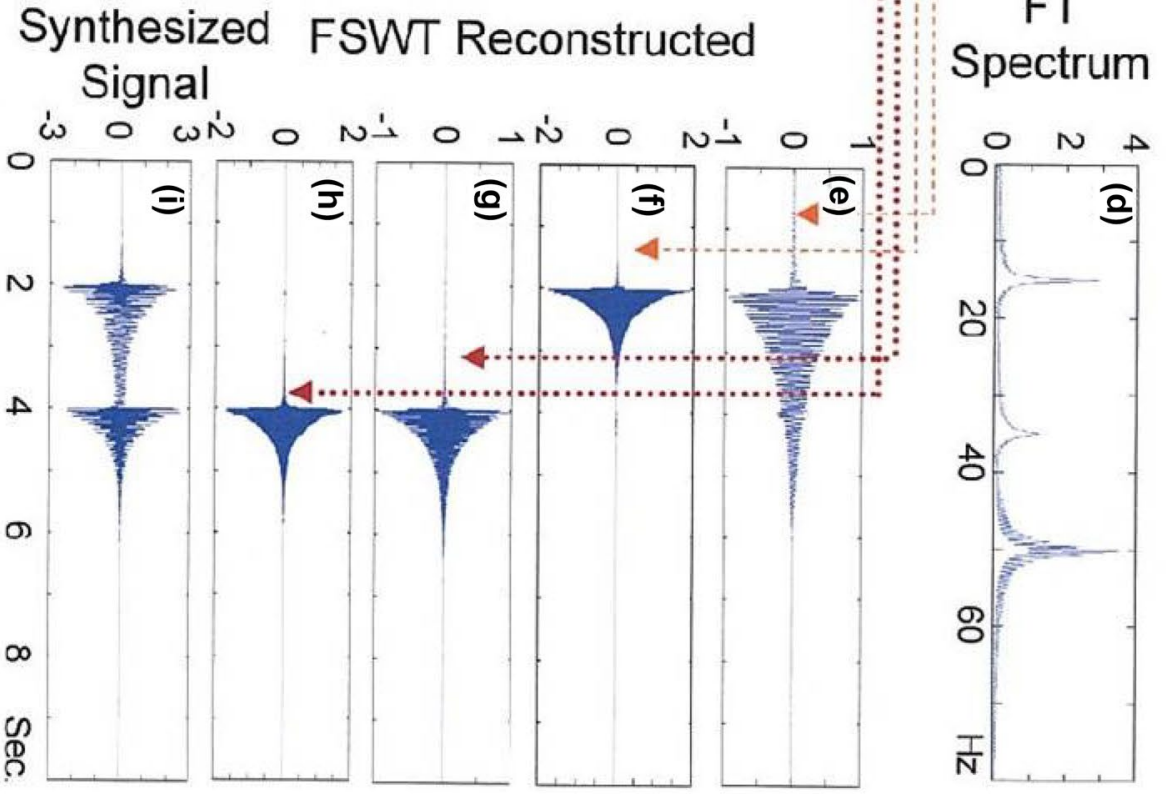

Comparison of Fig. 6e and $\mathrm{j}$ shows that regardless of noise, the analytical results obtained using the WVD do not show clear peaks in the time-frequency space.

These results indicate that compared with representative conventional time-frequency space analysis methods, namely, CWT (Morlet), STFT and WVD, the FSWTbased analysis method excels in showing clear peaks in the time-frequency space for vibration frequency (signal) and in resistance to noise. It is therefore evident that as a time-frequency space analysis method, the FSWT is more suitable for SRM than the other methods.

\section{Experimental studies}

The SRM scale concept was implemented to study the difference between two states of the system. The accuracy of detecting feature quantity differences when converting a parameter (structural parameter) for evaluating the condition (e.g., damage) of the bridge of interest (damage detection) to a probabilistic model by applying SRM that makes use of the above-mentioned characteristics of the FSWT is theoretically affected by the setting of the SRM scale parameter $\sigma$ [11]. This study, therefore, evaluated the effect of the scale parameter $\sigma$ on damage detection 


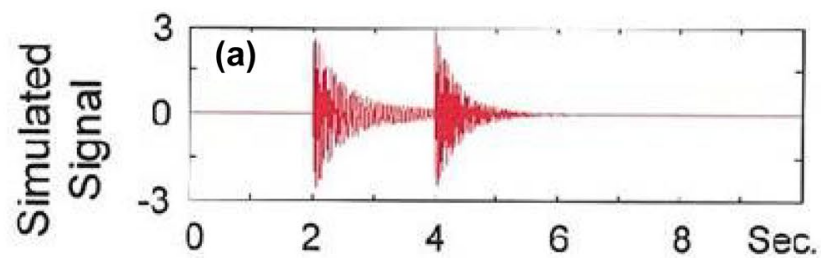

(b)
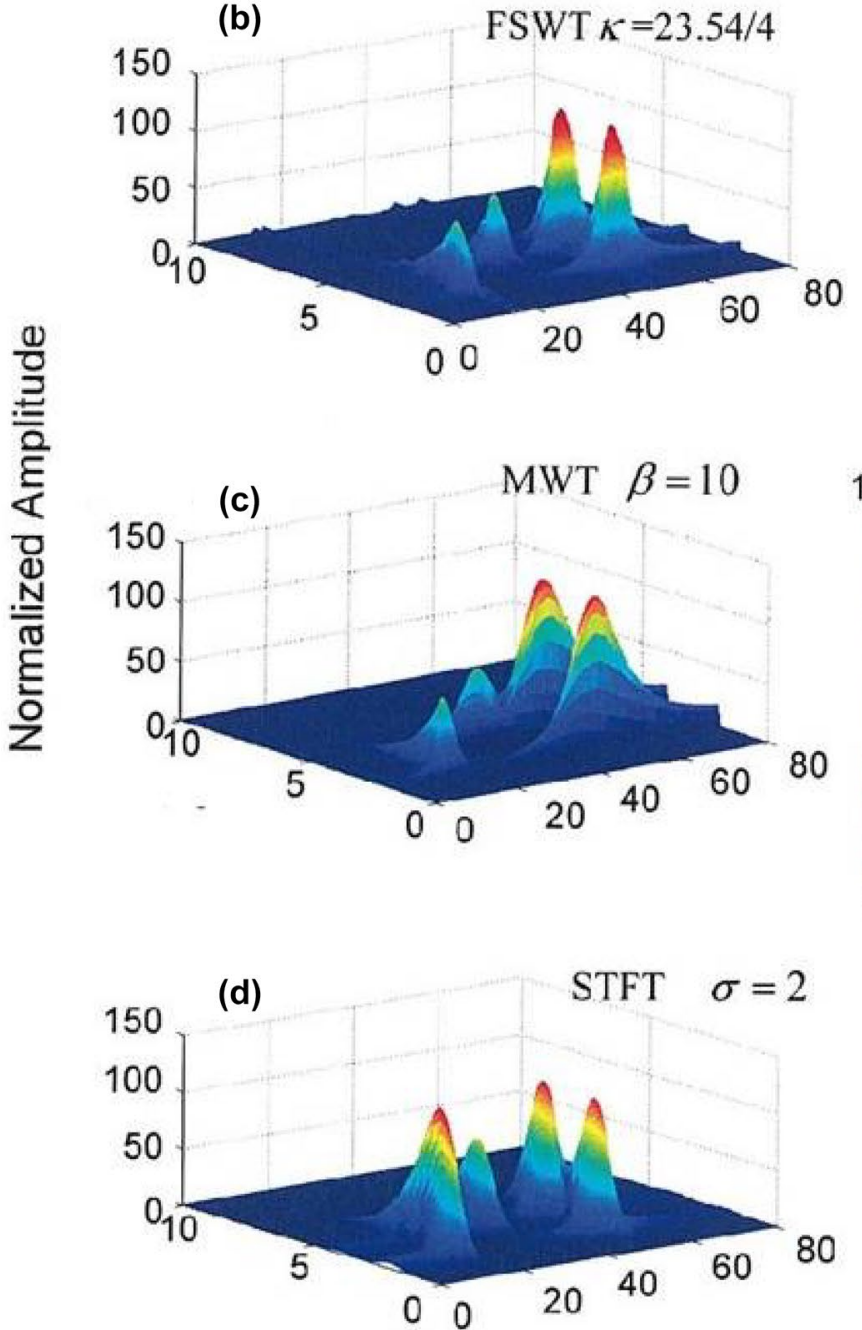

(e)

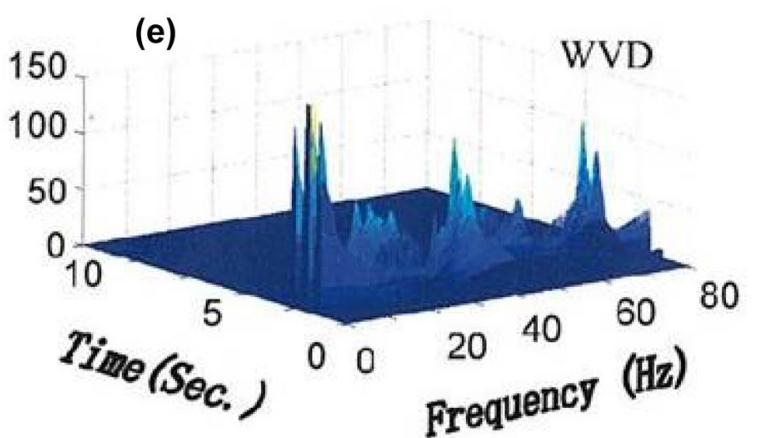

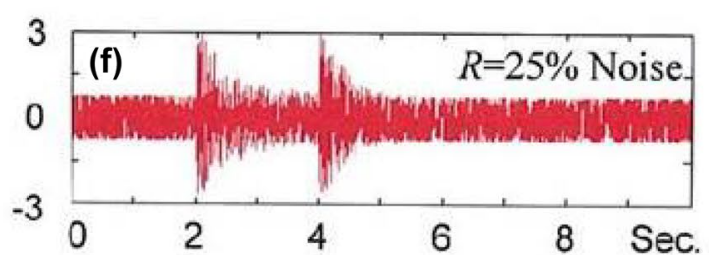

(g)

FSWT $\kappa=23.54 * 4$
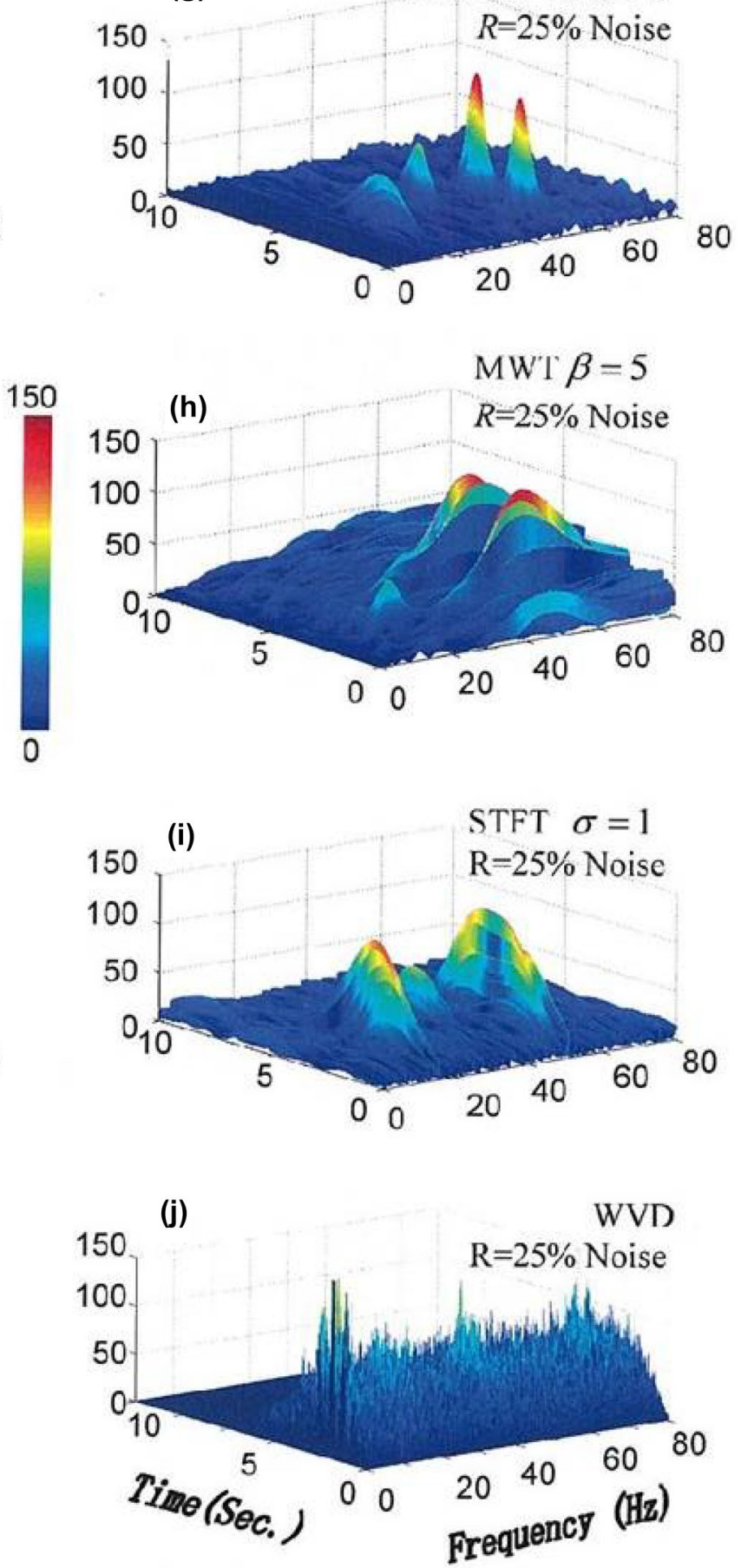

Fig. 6 Differences in time-frequency space analysis results obtained using two types of sample vibration data $[9,10]$ 
Table 1 Data on simulated waves

$\longrightarrow \quad$ : Weighted (probabilistically) output

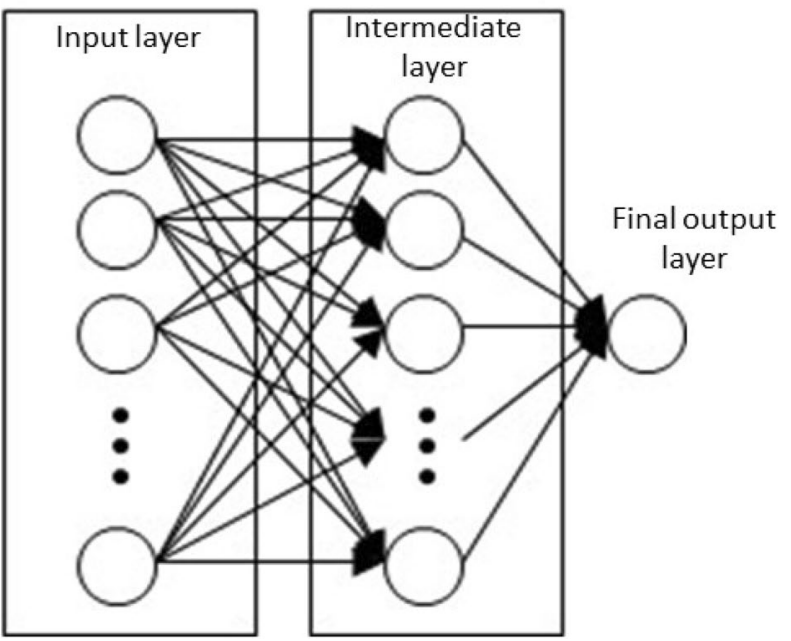

Fig. 7 Derivation of the equation of state using of a perceptron-type neural network

results using the results of two types of experiments carried out using simple bridge models with different types of known damage, namely, moving load experiments carried out using girders and impact hammer tests conducted using a bridge model consisting of three girders.

\subsection{Characteristics of SRM-based detection method}

Let $f(x)$ be a present state function of a structural system. Let $H^{n}$ be a finite feature Hilbert space assumed to be incorporated in the response $H^{\infty}$ of every structural system. Then, a feature vector expressing the present state of the structural system can be obtained as follows:

$x=\left(x_{1}(t), x_{2}(t), x_{3}(t), \ldots x_{n}(t)\right) \in H^{n} \subset H^{\infty}$

Here a probabilistic model of a perceptron-type neural network with an intermediate layer as shown in Fig. 7, giving an output of a certain probabilistic density from an arbitrary input vector, is regarded as an equation of state, and an arbitrary function can be obtained:

$\zeta=f(x) \in[0,1]$
SRM is the process in which this equation of state is determined from a given feature (input) vector (see Fig. 8). Since the state representation equation (SRE) thus determined can be defined in the finite feature Hilbert space, it can be derived by defining an arbitrary inner product function like Eq. (37):

$\zeta=f(\lambda, x)=\sum_{i}^{m} \lambda_{i} k\left(x_{i}, x\right)$

Thus, the problem can be reduced to the problem of minimization and optimization using Eq. (37) as a variable. In Eq. (37), $k\left(x_{i}, x\right)$ is called a kernel function, $k(\cdot, \cdot)$. Many kernel functions have been proposed, such as the d-degree polynomial kernel, the radial basis function kernel and the sigmoid kernel [10]. For the purposes of this study, the Gaussian kernel shown in Eq. (38) is used for SRM:

$k\left(x_{i}, x\right)=\exp \left(-\frac{d\left(x_{i}, x\right)}{\sigma^{2}}\right)$

Following the setting of the SRM scale parameter $\sigma$, the kernel function of Eq. (38) is determined, and the minimization-optimization problem expressed by Eq. (37) is solved using the gradient method (see Fig. 8). In Eq. (38), $\sigma$ is a scale parameter to be determined by the user as appropriate within the range $0<\sigma \leqq 0.5$.

\subsection{Test description}

\subsubsection{Moving load experiment}

Two types of verification tests using bridge model girders were carried out: single-girder moving load experiments and impact hammer tests using a bridge model girder structure consisting of three girders.

Figure 9 illustrates the girder used for the single-girder moving load experiment. In the single-girder moving load experiment, a moving load designed to simulate a vehicle was made to move on a simply supported girder, and vertical acceleration was measured at three points located along the girder (see Fig. 9). Seven types of girders were prepared for the single-girder experiments: an undamaged wideflange steel beam and six types of steel beams with different degrees of artificially introduced lower-flange damage (stiffness reduction) (see Figs. 10, 12).

\subsubsection{Impact hammer test}

In the impact hammer test conducted using a three-girder bridge model, accelerometers were installed on the bridge model girder structure shown in Fig. 11, and vibrations were measured at nine points on the bridge model girder 


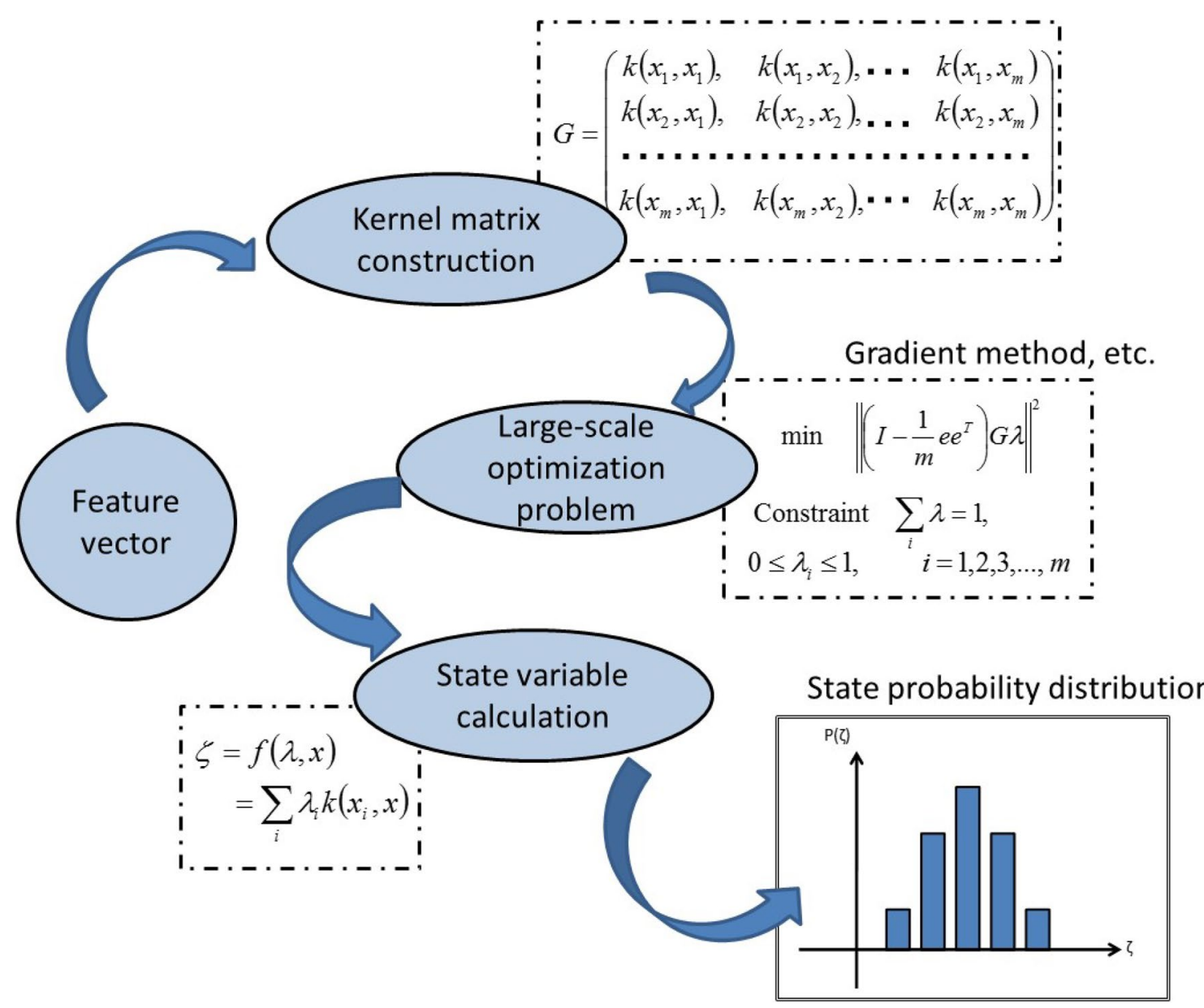

Fig. 8 Process to determine the equation of state (state probability distribution) from a given feature vector

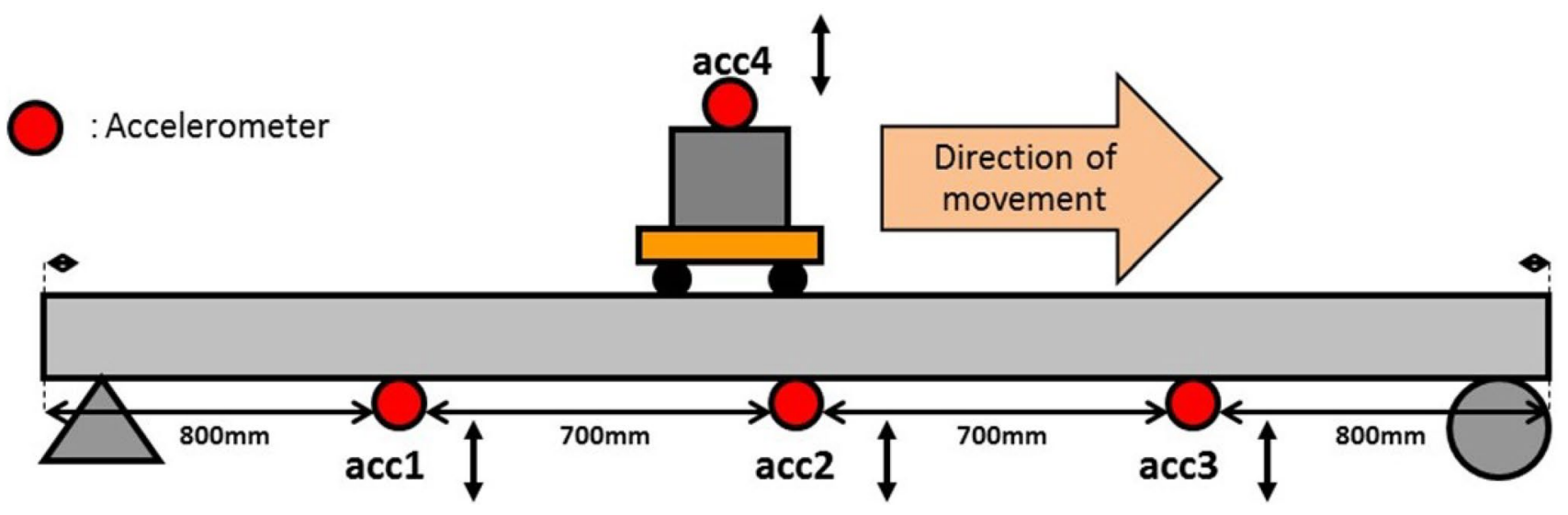

Fig. 9 Moving load experiment using a single-girder specimen

structure by installing accelerometers and exciting the girder structure with a hammer impact. Figures 12 and 10 (shown earlier) show information on the undamaged members and artificially damaged members of the bridge model girder structure used, including their details such as the degrees and locations of damage. Figure 13 illustrates the bridge model girder structure used in the experiments and the locations of the nine accelerometers. In the experiments, 

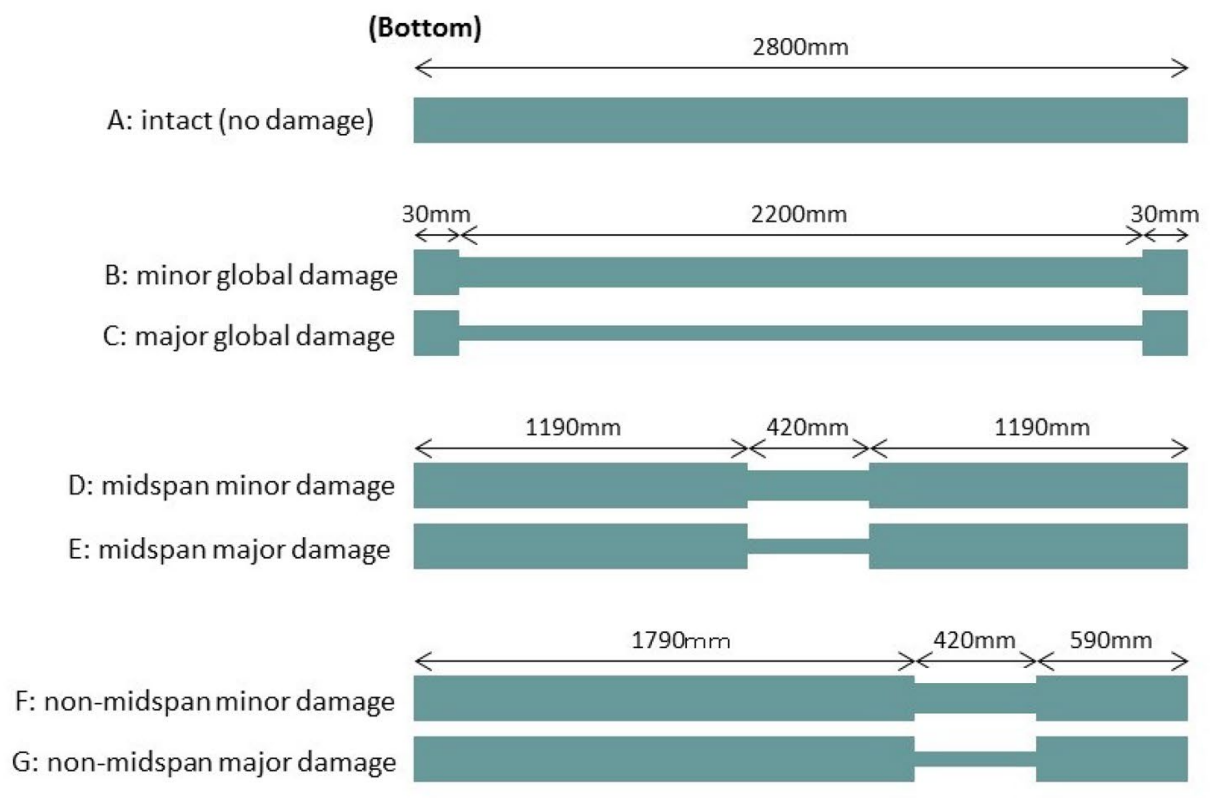

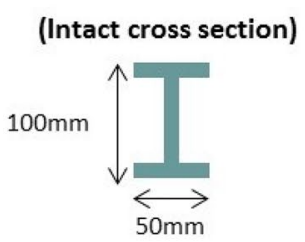

(Cross section with minor damage)

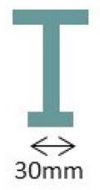

(Cross section with major damage)

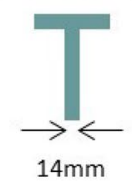

Fig. 10 Dimensions of intact members and artificially damaged members (artificial damage given to lower flange)

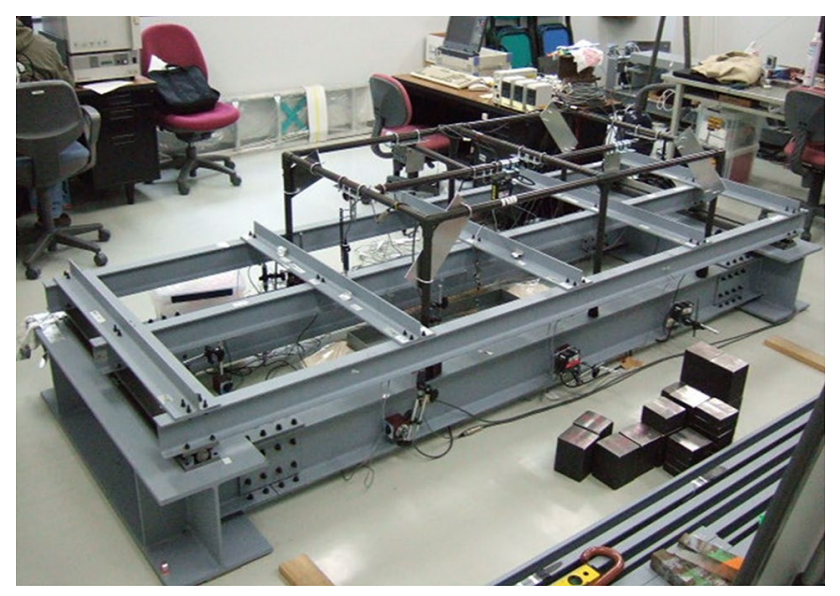

Fig. 11 General view of bridge model girder structure consisting of three girders $[9,10]$

damaged girder locations were changed among Girders 1, 2 and 3. For each of those locations, tests were conducted for no damage, minor damage (about 20\% stiffness reduction) and major damage (about $40 \%$ stiffness reduction) cases and the damage location combinations shown in Fig. 10. For each of those combinations, a hammer impact was given at a total of nine locations (intersections B1 to D3 of Cross Beams 1, 2 and 3 and Girders 1,2 and 3) to vibrate the bridge model girder structure, and vertical acceleration data were recorded. This experiment was carried out ten times at each location. The sampling rate was $1000 \mathrm{~Hz}$, and the measurement (sampling) time was $15-20 \mathrm{~s}$ for the moving load test and $5 \mathrm{~s}$ for the impact hammer test.

\subsection{Discussion}

Figure 14 shows examples of acceleration waveforms observed in the moving load experiment and the impact hammer test, and Fig. 15 shows the FSWT-based time-frequency space analysis results. The analytical results obtained by the FSWT show frequency $(\mathrm{Hz})$ (in spatial representation, frequency and time) on the horizontal axis, time (s) (in spatial representation, amplitude) on the vertical axis, and the brightness of the color represents power.

To evaluate the state of the bridge model girder structure by applying SRM, it is necessary to calculate the coefficient $\alpha$ (damping parameter) in the amplitude envelope $x=A e^{-\alpha t}$ (see Fig. 4) and use it as a feature quantity. By applying the modal damping function (MDF) to the data obtained through FSWT-based conversion, the coefficient $\alpha$ can be calculated for any time-frequency range. This characteristic is used to define the feature extraction range in terms of feature blocks (FB) and construct feature vectors. This segmentation approach makes it possible to deal with changes in natural frequency by the FSWT, which is implemented to transform the time domain into the time-frequency domain.

Figures 16 and 17 show FSWT-converted time-frequency space data (see Figs. 14, 15) divided into 80 and 100 blocks, which were obtained by dividing the converted time-frequency space data into 20 blocks along the frequency axis and four blocks (moving load experiment) or five blocks (impact hammer test) along the time axis. The steps for the feature extraction algorithm were as follows: 


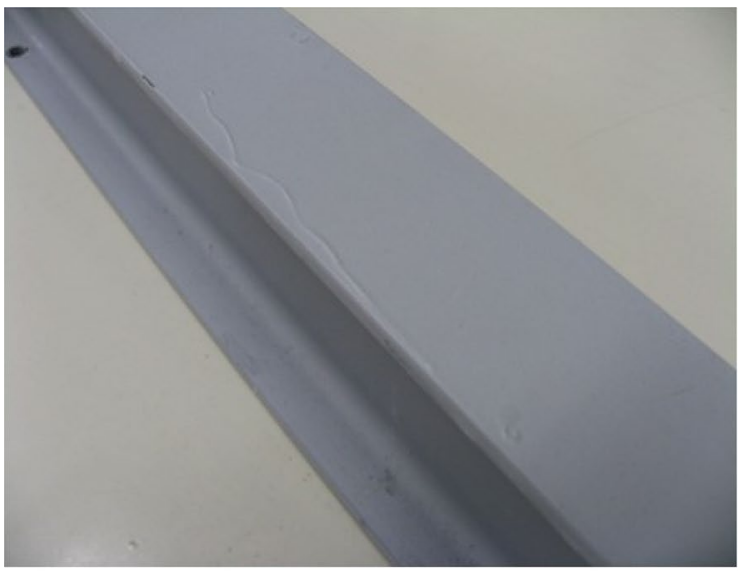

(a) Intact member

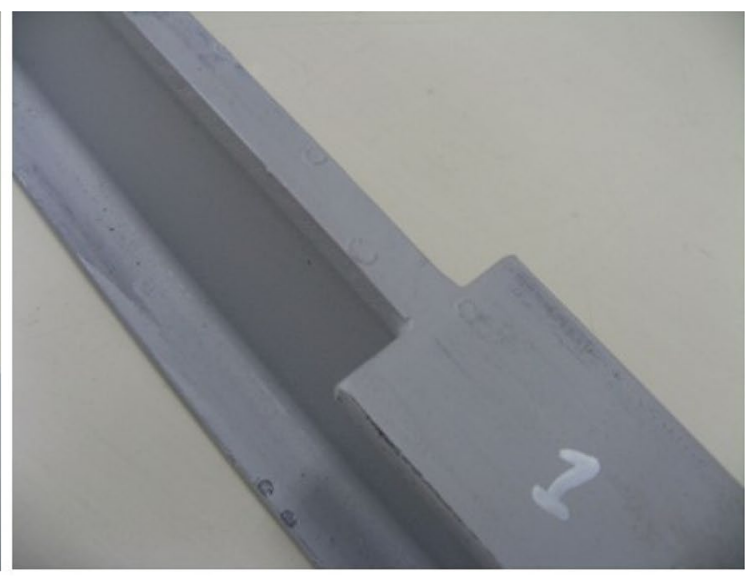

(b) Damaged member

Fig. 12 Intact and damaged members of bridge model girder structure (lower flange of girder) $[9,10]$

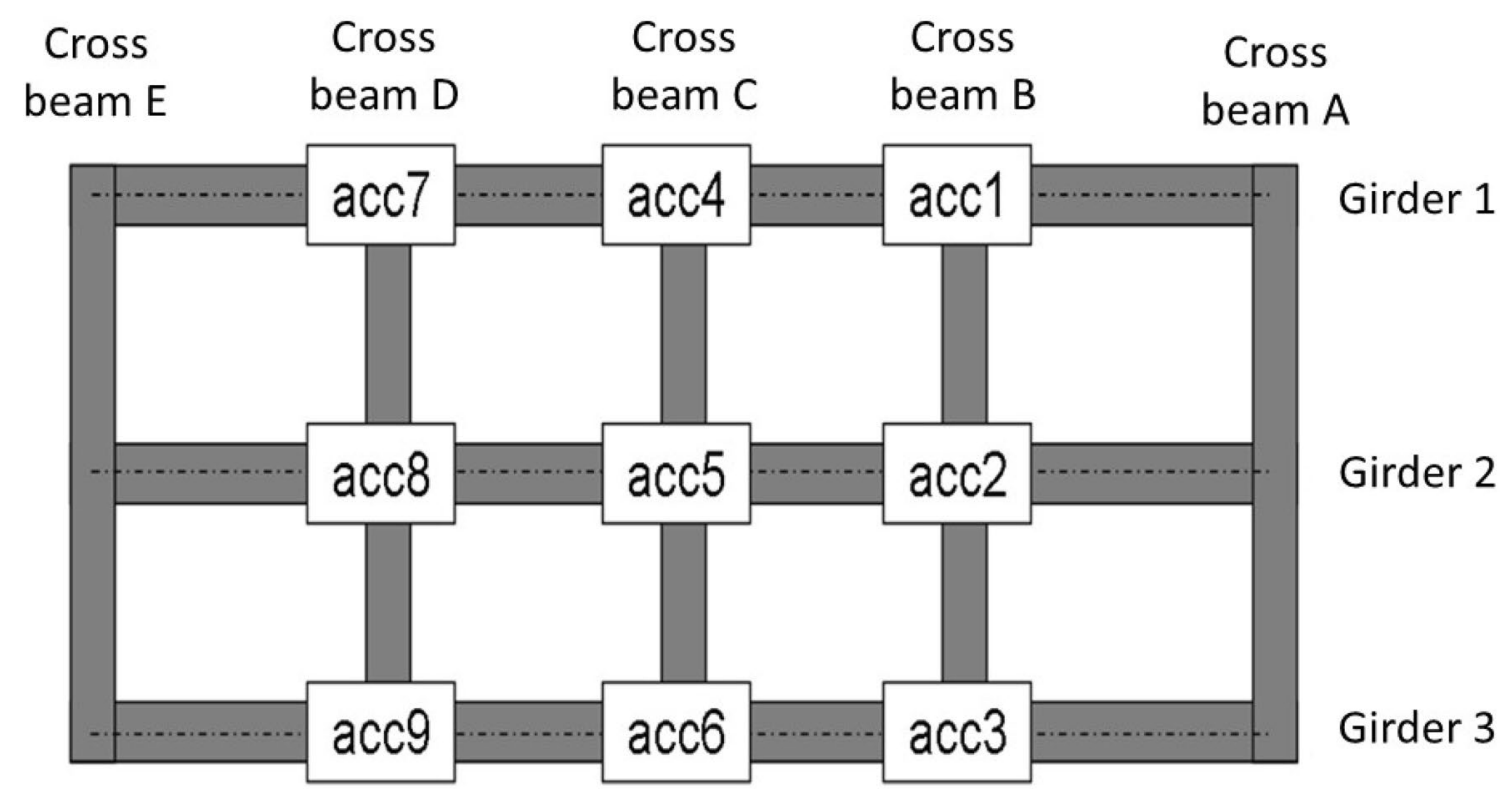

Fig. 13 Configuration of three-girder bridge model girder structure and locations of accelerometers

STEP 1: Compute the FSWT expression for each sensor response, and denote it by $W_{i}(t, \omega, \alpha), i=1,2, \ldots N_{s}$, where $N_{s}$ is the number of sensors.

STEP 2: Take the maxima of $\left|W_{i}(t, \omega, \kappa)\right|$, i.e., $|W|_{M}=\max _{t \geq 0, \omega \geq 0, i}\left|W_{i}(t, \omega, \kappa)\right|$ and record $\left(t_{M}, \omega_{M},|W|_{M}\right)$ as a trigger condition.

STEP 3: Define the frequency feature sampling line (FSL) and time feature sampling line (TSL), and record $M_{f}$, the total number of FSLs, and $M_{t}$, the total number of TSLs. The small block is called a feature block (FB), as shown in Figs. 16 and 17.

STEP 4: Compute the modal damping feature at each feature block as

$$
\begin{aligned}
\alpha_{i p q} & =\left.\operatorname{MDF}\left(\left|W_{i}(t, \omega, \kappa)\right|\right)\right|_{(t, \omega) \in \mathrm{FB}_{\mathrm{pq}},} \\
i & =1,2, \ldots N_{s}, p=1,2 \ldots M_{f}, q=1,2 \ldots M_{t}
\end{aligned}
$$



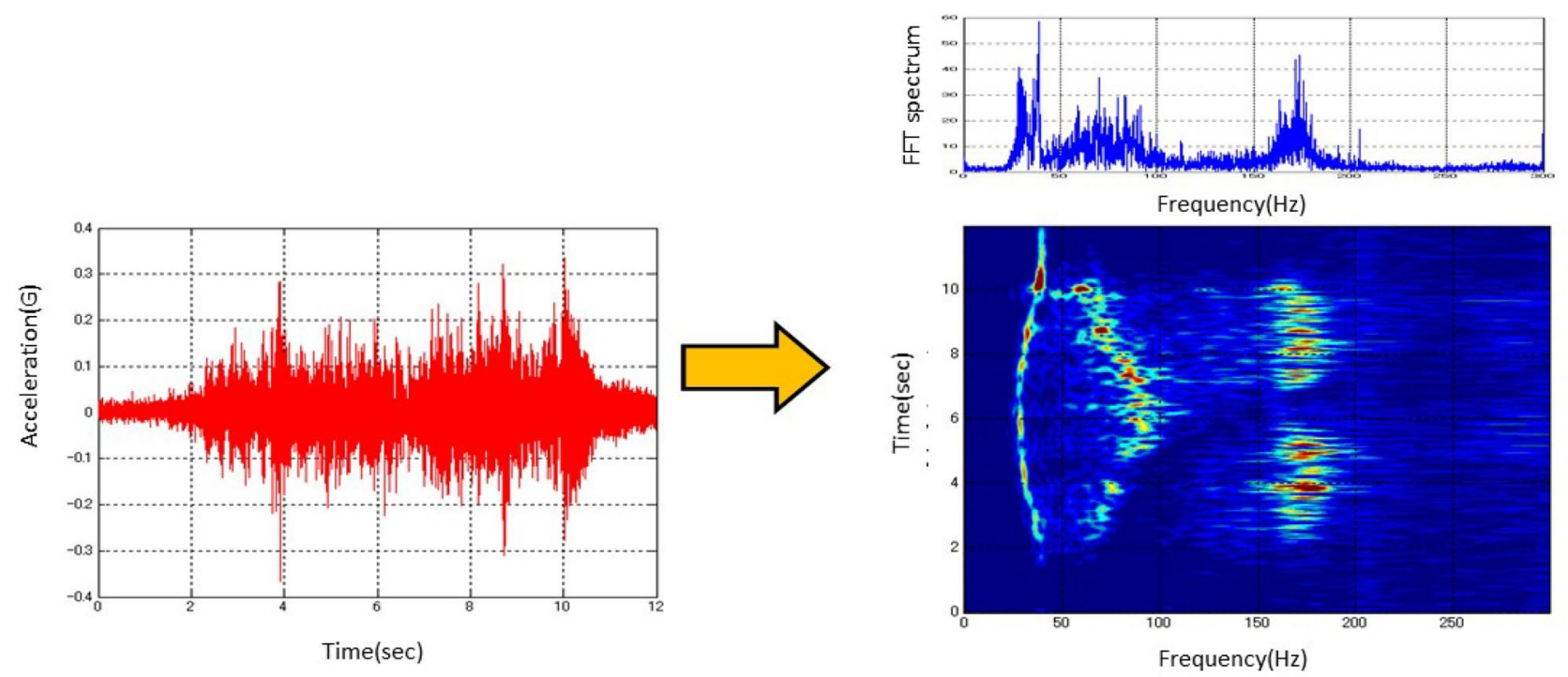

(a) Acceleration waveform

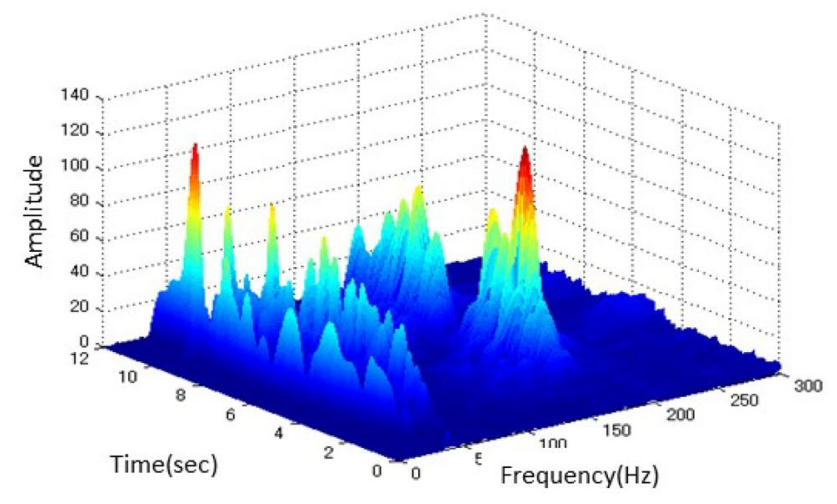

(b) Time-frequency space analysis

Fig. 14 Examples of acceleration waveform obtained from moving load experiment and time-frequency space analysis results obtained by FSWT

STEP 5: Define: $V_{i}=\left(W_{M 1}, \alpha_{i p g}\right) i=1,2, \ldots N_{s}, p=1$, $2 \ldots M_{f}, q=1,2 \ldots M_{t}$, where

$W_{i p q}=\left.E\left(\left|W_{i}(t, \omega, \kappa)\right|\right)\right|_{(t, \omega) \in \mathrm{FB}_{\mathrm{pq}}}$

where $E(\cdot)$ means the average value, and $V_{i}$ is the time-frequency feature (TFF) vectors of the system.

Here, a real experiment using the laboratory bridge monitoring system is considered. Let $N_{s}=11$, $M_{f}=300, M_{t}=3$, and there are 1200 features for each sensor. Thus, the acceleration sensor data contains data for 9 hammer impacts $\times 11$ acceleration sensors $\times 10$ test iterations $=990$ groups, and there are $990 \times 1200=1.1 \mathrm{M}$ features, which is a big number. This is an obvious reason why the SRM is a large-scale problem.

Feature quantities are extracted by applying Eq. (41) to the feature blocks thus defined, and the results are substituted in an MDF. The coefficient $\alpha$ in the amplitude $x=A e^{-\alpha t}$ envelope obtained by arbitrary domain FSWT was calculated at multiple locations, and the coefficients thus determined were used as feature vectors for damage detection. The first step in calculating the coefficient $\alpha$ is the calculation using Eq. (41) when $t \in[0, T] \subset(0,+\infty)$ :

$S\left(x^{n}\right)=\frac{1}{T} \int_{0}^{T} x^{n} \mathrm{~d} t, \quad n=0.5,1,2,4$ 


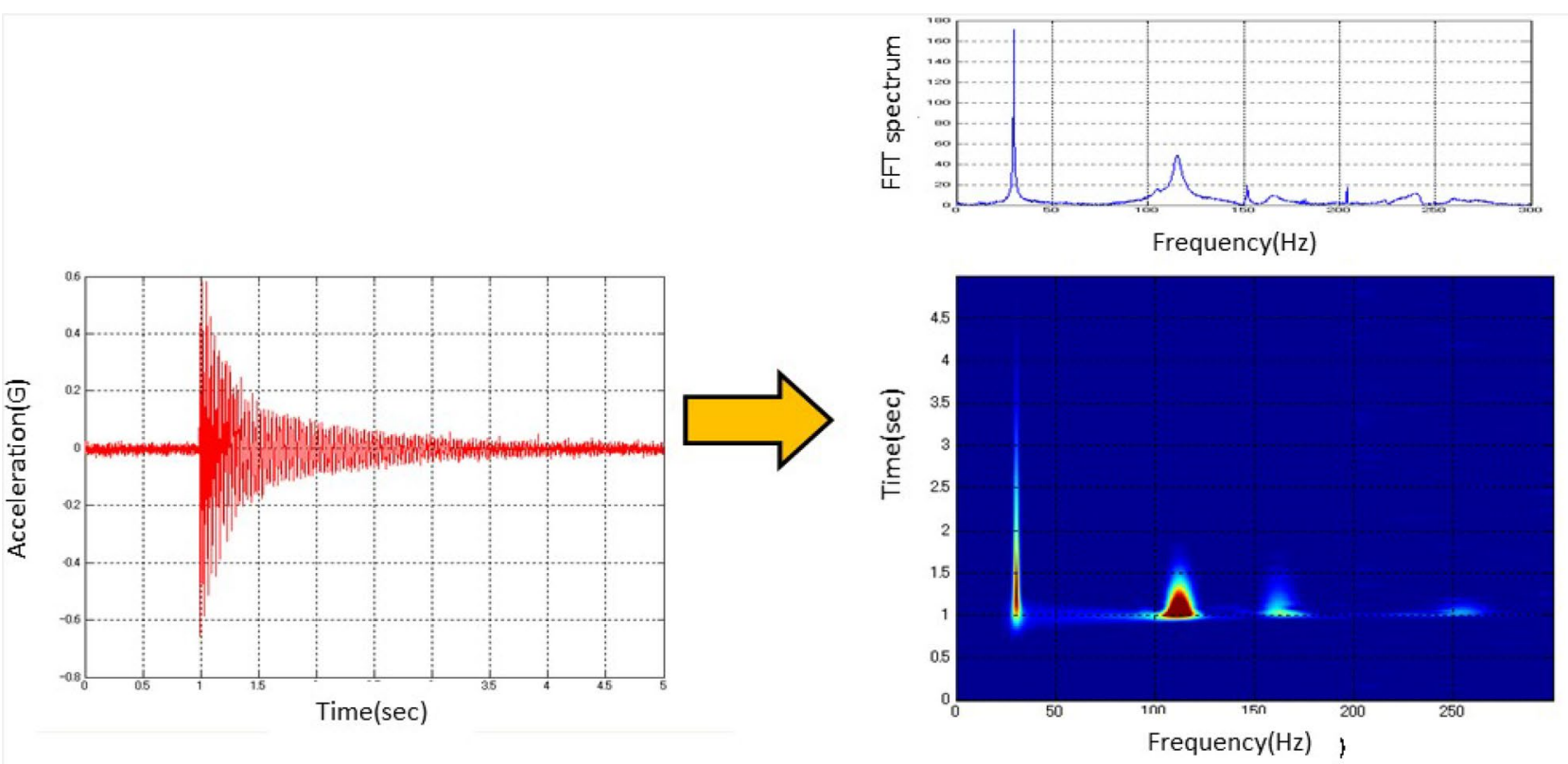

(a) Acceleration waveform

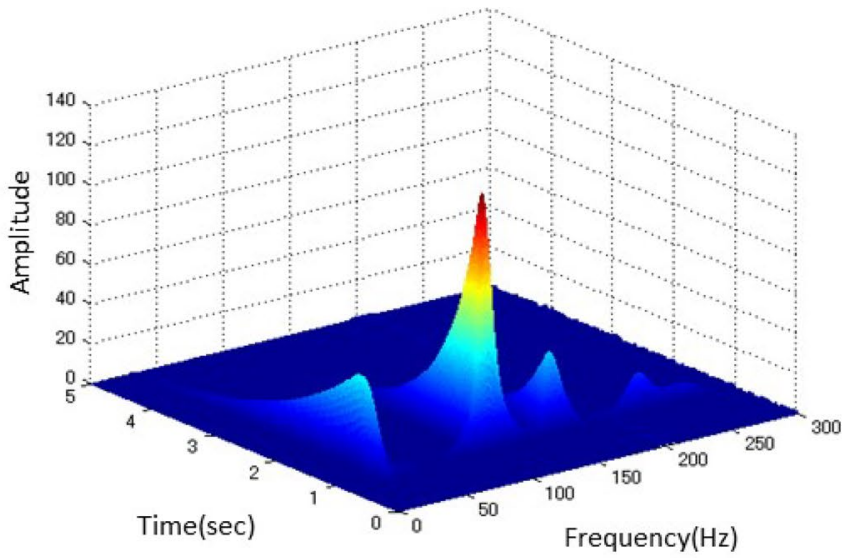

(b) Time-frequency space analysis

Fig. 15 Examples of acceleration waveform obtained from impact hammer testing and time-frequency space analysis result obtained by FSWT $[9,10]$

The calculation of Eq. (41) is easy, and the calculated value, $S\left(x^{n}\right)$ thus obtained is substituted in Eq. (42) to calculate the coefficient $\alpha$ :

$\alpha=4 \sqrt{S^{2}(\sqrt{x}) S\left(x^{2}\right)-S^{3}(x)} /\left(S^{2}(\sqrt{x}) \sqrt{S\left(x^{2}\right)}\right) / T$

In this study, the value of $\alpha$ for each feature block calculated from the measurement data obtained from the experiments carried out for all combinations mentioned earlier was used as an input, and a state probability distribution model (probabilistic model) was constructed using SRM.
Figures 18 and 19 show examples of calculated block-byblock values of damping parameter $\alpha$ for the bridge model girder structure in a sound condition obtained from the moving load experiment and the impact hammer test, respectively. These results indicate that feature quantities in a block increase when there is a peak frequency in the block which represents the damping characteristics of a multi-model signal by a frequency slice algorithm.

Two values of the SRM scale parameter $\sigma$, namely, $\sigma=1 / 2$ and $\sigma=1 / 8$, were examined in evaluating the effects of different scale parameter values on damage detection results. Also, SRM-based state variable values determined by following the steps shown in Fig. 8 were compared to examine 


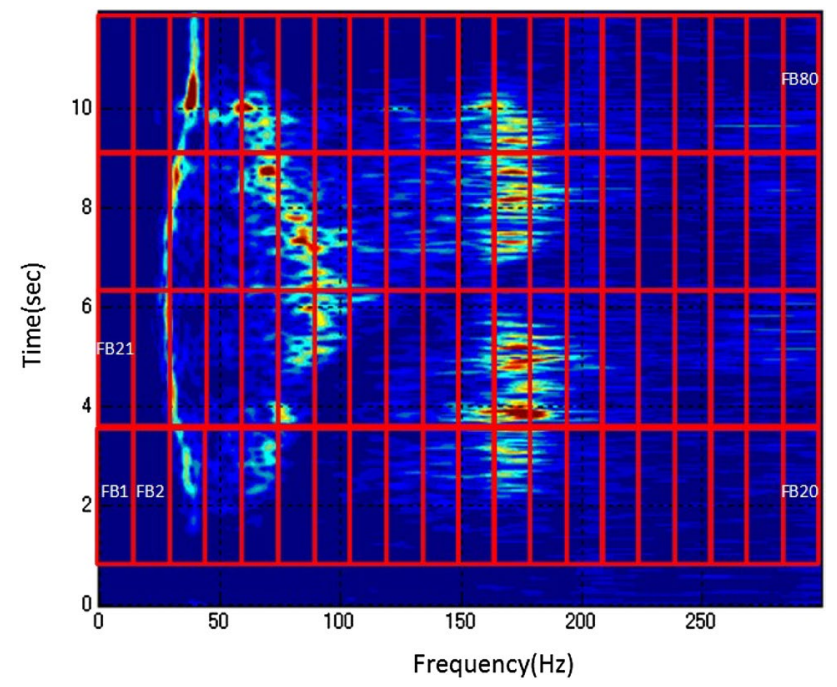

Fig. 16 Examples of blocks in time-frequency space defined for moving load experiment

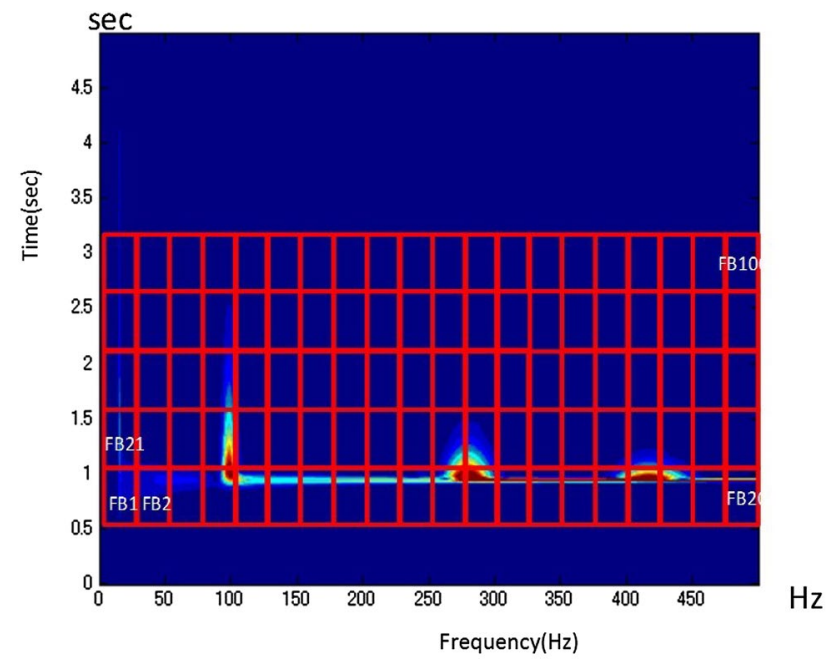

Fig. 17 Examples of blocks in time-frequency space defined for impact hammer testing $[9,10]$

how the state probability distribution changes in the nodamage girder cases and damaged girder cases, respectively.

Figure $20 \mathrm{a}$ and $\mathrm{b}$ compares the state probability distributions ( $\sigma=1 / 2$ and $\sigma=1 / 8)$ in the no-damage girder case and the major global damage case (Type $\mathrm{C}$ member) (see Fig. 10) observed in the moving load experiments conducted using separate girders. These results do not indicate any significant differences due to the SRM scale, but they do show that the histogram tends to shift leftward and concentrate as damage grows. Figure $21 \mathrm{a}$ and $\mathrm{b}$ compare the state probability distributions of the no-damage three-girder bridge model and the damaged three-girder bridge model in which

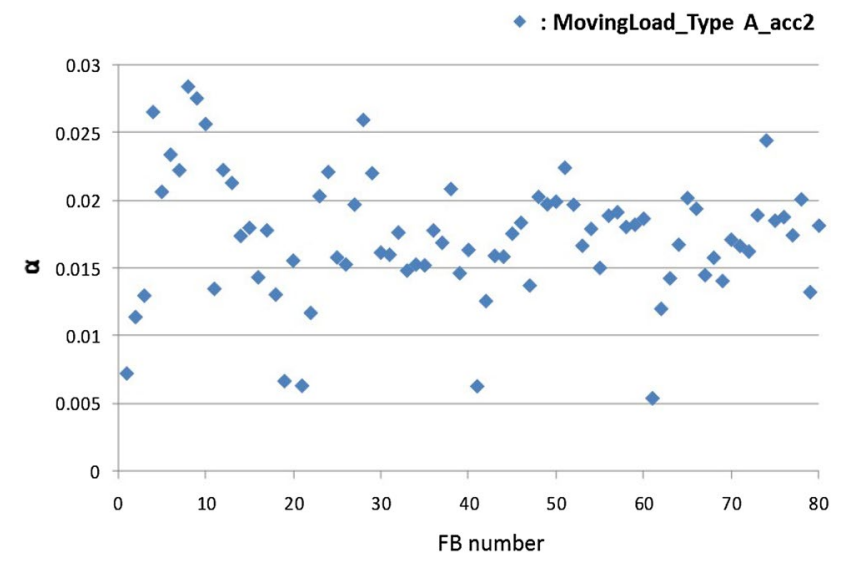

Fig. 18 Example of single-girder damping parameter extraction in moving load experiment [intact member (Type A member)]

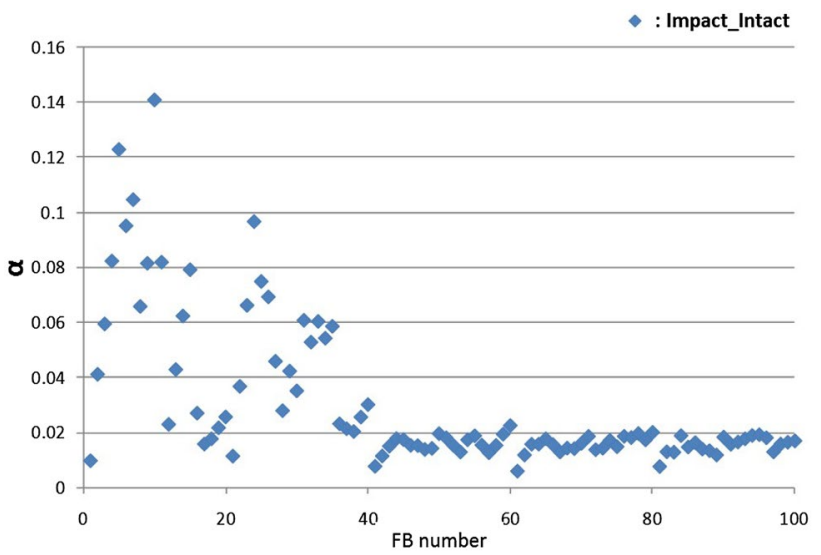

Fig. 19 Example of damping parameter extraction from intact bridge model girder structure in impact hammer test

a Type $\mathrm{C}$ member is used as Girder $2((1) \sigma=1 / 2,(2) \sigma=1 / 8)$ in the form of histograms like the results of the moving load experiment. These results indicate that at $\sigma=1 / 2$ the histogram does not show any significant differences in changes although relatively heavy damage was anticipated, while at $\sigma=1 / 8$ the histogram shows significant differences due to damage.

As these analytical results indicate, since the Gaussian kernel scale parameter for SRM greatly affects the detection accuracy, the quality of detection results is adversely affected if a large value like $\sigma=1 / 2$ is used. The reason is thought to be that as the value of the SRM scale parameter $\sigma$ increases, the distribution of probability densities output to the probability variable becomes more or less flattened so that the feature necessary for detection accuracy becomes less discernible. It is therefore necessary to use a more or less focused SRM scale parameter for the probability variable expressing a damage-related feature quantity. 


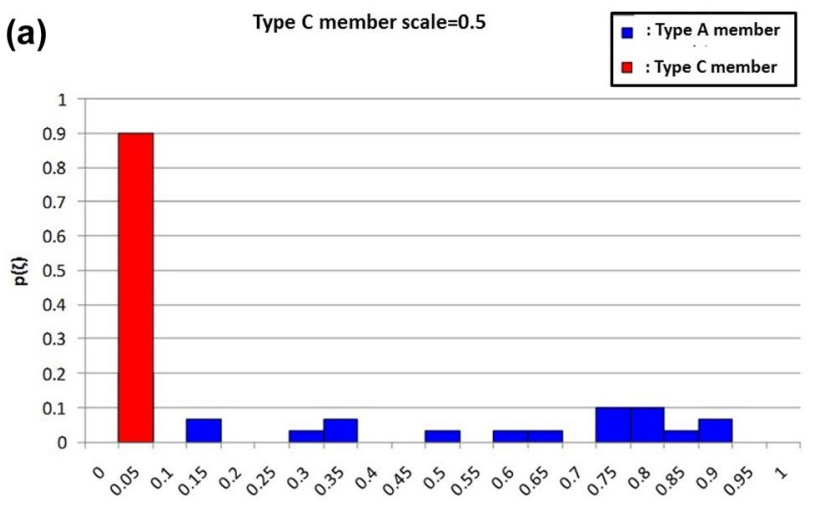

$\zeta$

(b)

Type $\mathrm{C}$ member scale $=\mathbf{0 . 1 2 5}$
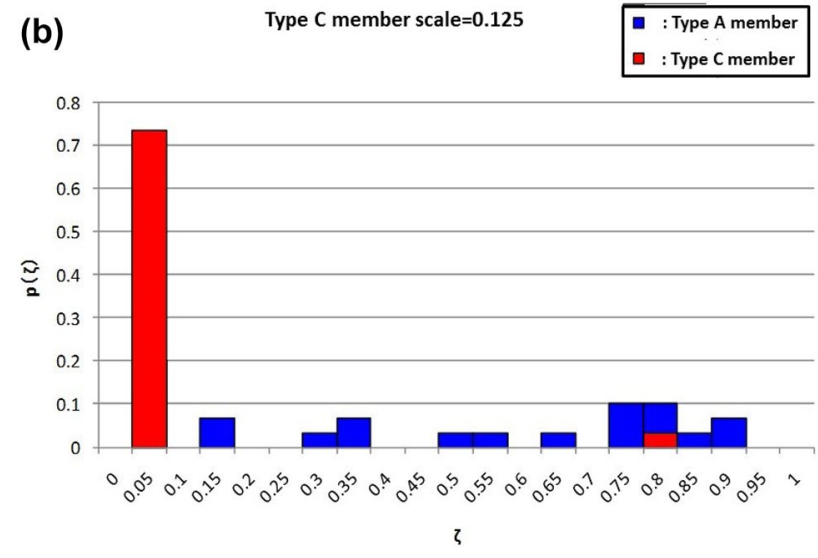

Fig. 20 a State probability density distribution (histogram) at $\sigma=1 / 2$ : no damage versus major global damage (Type $\mathrm{C}$ member) (singlegirder case, moving load experiment). b State probability density distribution (histogram) at $\sigma=1 / 8$ : no damage versus major global damage (Type $\mathrm{C}$ member) (single-girder case, moving load experiment)

\section{Conclusions}

In this study, a new method for damage detection (performance evaluation) from a large amount of continually collected measurement data by applying the State Representation Methodology (SRM) was proposed, and a number of time-frequency analysis methods necessary for the implementation of the proposed method were compared and discussed. As part of the study, the SRM scale parameter, which can affect damage detection accuracy, was also evaluated experimentally.

The results obtained from this study are as follows:

1. Using sample vibration data, a comparison was made of a number of representative time-frequency analysis techniques, including the Frequency Slice Wavelet Transform (FSWT), the short-time Fourier transform (STFT), the continuous wavelet transform (CWT) and the Wigner-Ville distribution (WVD). The comparison showed that the FSWT makes it possible to accurately
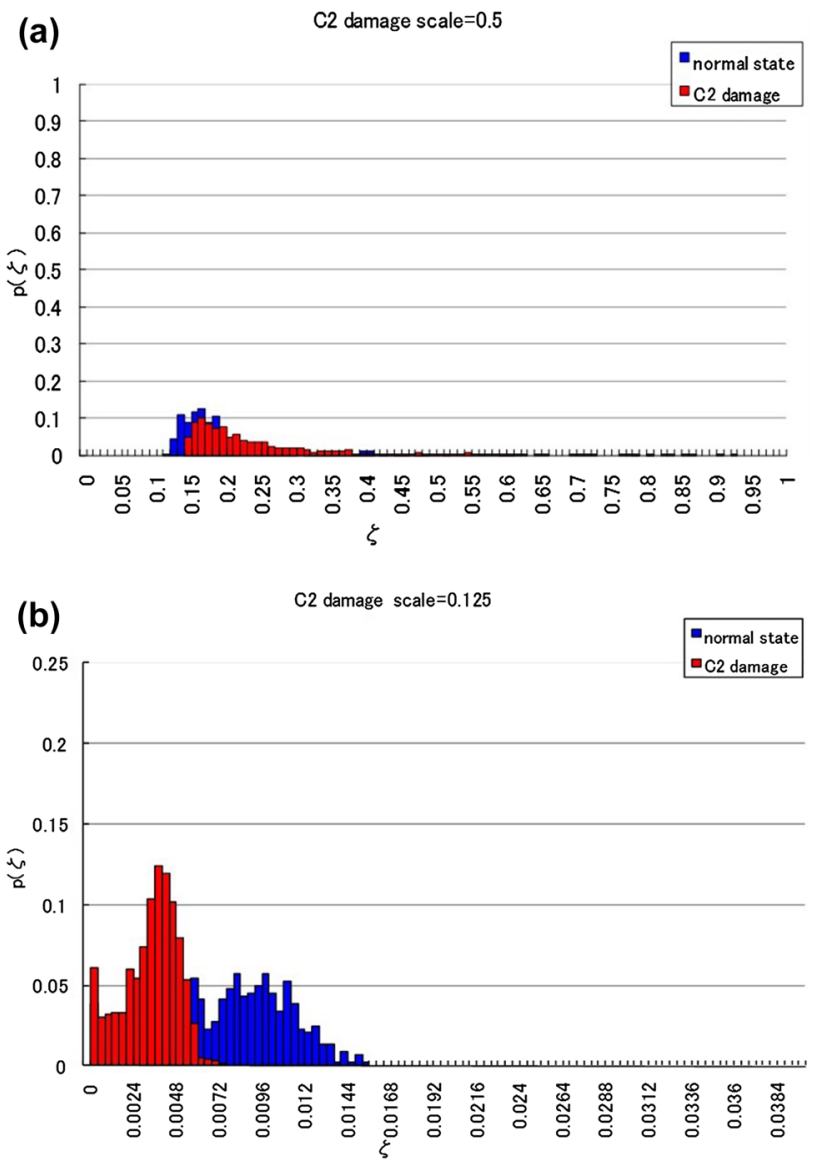

Fig. 21 a State probability density distribution (histogram) at $\sigma=1 / 2$ : no damage case versus Type-C-member-for-Girder-2 case (impact hammer test using a three-girder bridge model). b State probability density distribution (histogram) at $\sigma=1 / 8$ : no damage case versus Type-C-member-for-Girder- 2 case (impact hammer test using a threegirder bridge model)

extract peaks in the time-frequency space and is superior to the other methods in resistance to noise. The FSWT, therefore, is more suitable than the other methods for use with SRM.

2. When SRM is used, the SRM scale parameter $\sigma$ of the Gaussian kernel affects the damage detection accuracy. If the SRM scale parameter is not properly set to deal with the input vector variability, the detection accuracy may be adversely affected. To realize high-accuracy damage detection by use of the SRM, it is necessary to use a setting for $\sigma$ of at least about $1 / 8$.

3. The state representation methodology (SRM) is intended for a non-parametric description of a system state described by state variables. The state variables are calculated by the state representation equation (SRE) to express a steady state of the system. The SRM is a useful method for bridge condition assessment in structural health monitoring (SHM). 
Open Access This article is distributed under the terms of the Creative Commons Attribution 4.0 International License (http://creativeco mmons.org/licenses/by/4.0/), which permits unrestricted use, distribution, and reproduction in any medium, provided you give appropriate credit to the original author(s) and the source, provide a link to the Creative Commons license, and indicate if changes were made.

\section{References}

1. Miyamoto A (2009) Usage management of civil structures. In: Boller C, Chang F, Fujino Y (eds) Encyclopedia of structural health monitoring, vol 4. Wiley, London, pp 1635-1671

2. Furuta H, Kayano M, Watanabe E (2007) Current status and future issues on bridge maintenance and bridge management system. J Jpn Soc Civ Eng F 63(3):287-294

3. Yanev B (2007) Bridge management. Wiley, New Jersey

4. Miyamoto A, Isoda S (2012) Sensitivity analysis of mechanical behaviors for bridge damage assessment. Struct Eng Mech 41(4):539-558

5. Worden K, Lane AJ (2001) Damage identification using support vector machines. Smart Mater Struct 10:540-547

6. Bridge vibration monitoring sub committee of JSCE committee of structural engineering (2000). Guideline for bridge vibration monitoring. Structural Engineering Series 10, Japan Society of Civil Engineers (JSCE), p 1-8

7. Kawamura S, Okabayashi T, Takagi S (2000) Development of remote measurement system for bridge vibration by mobile communication. J Struct Eng A JSCE 46A:539-546

8. Nagai N, Mita A, Yakoh T, Sato T (2003) Wireless sensor for structural health monitoring. J Jpn Assoc Earthq Eng JSCE $3(4): 1-13$
9. Yan Z-H, Miyamoto A, Jiang Z-W (2009) Frequency slice wavelet transform for transient vibration response analysis. Mech Syst Signal Process 23(5):1474-1489

10. Yan Z-H, Miyamoto A, Jiang Z-W, Liu X-L (2010) An overall theoretical description of frequency slice wavelet transform. Mech Syst Signal Process 24(2):325-572

11. Yan Z-H, Miyamoto A (2009) State representation methodology (SRM) and its application to bridge condition assessment, lifetime management book no. 9. The Research Center for Environmental Safety, Yamaguchi University, Yamaguchi

12. Miyamoto A (2013) A new damage detection method for bridge condition assessment in structural health monitoring. J Civ Struct Health Monitor 3(4):269-284

13. Chui CK, Wang JZ (1997) A study of asymptotically optimal time-frequency localization by scaling function and wavelet. Ann Numer Math 4:193-216

14. Yan Z, Miyamoto A (2008) An idea of state representation methodology-theory and application. Strateg Lifetime Manag Civ Infrastruct Latest Inf Technol 1:21-47

15. Cristianini N, Shawe-Tayor J (2000) An introduction to support vector machines and other kernel-based learning methods. Cambridge University Press, Cambridge

Publisher's Note Springer Nature remains neutral with regard to jurisdictional claims in published maps and institutional affiliations. 